\title{
A fractional Yamabe flow and some applications
}

\author{
Tianling Jin and Jingang Xiong
}

November 8, 2018

\begin{abstract}
We introduce a fractional Yamabe flow involving nonlocal conformally invariant operators on the conformal infinity of asymptotically hyperbolic manifolds, and show that on the conformal spheres $\left(\mathbb{S}^{n},\left[g_{\mathbb{S}^{n}}\right]\right)$, it converges to the standard sphere up to a Möbius diffeomorphism. These arguments can be applied to obtain extinction profiles of solutions of some fractional porous medium equations. In the end, we use this fractional fast diffusion equation, together with its extinction profile and some estimates of its extinction time, to improve a Sobolev inequality via a quantitative estimate of the remainder term.
\end{abstract}

\section{Introduction}

Let $\left(M, g_{0}\right)$ be a compact Riemannian manifold of dimension $n \geq 2$. The following evolution equation for the metric $g$

$$
\frac{\partial}{\partial t} g(t)=-\left(R_{g(t)}-r_{g(t)}\right) g(t), \quad g(0)=g_{0}
$$

was introduced by Hamilton in [44], and is known as the Yamabe flow. Here, $R_{g(t)}$ is the scalar curvature of $g(t)$ and $r_{g(t)}=\operatorname{vol}_{g(t)}(M)^{-1} \int_{M} R_{g(t)} \mathrm{d} v o l_{g(t)}$ is the average of $R_{g(t)}$. The existence and convergence of solutions of (1) were established through [44], [25], [76], [70], [13] and [15].

In [41], Graham, Jenne, Mason and Sparling constructed a sequence of conformally invariant elliptic operators, $\left\{P_{k}^{g}\right\}$, on $(M, g)$ for all positive integer $k$ if $n$ is odd, and for $k \in\{1, \cdots, n / 2\}$ if $n$ is even. Moreover, $P_{1}^{g}$ is the conformal Laplacian $L_{g}:=-\Delta_{g}+c(n) R_{g}$ and $P_{2}^{g}$ is the Paneitz operator. The construction in [41] is based on the ambient metric construction of [35]. Up to a positive constant $P_{1}^{g}(1)$ is the scalar curvature of $g$ and $P_{2}^{g}(1)$ is the $Q$-curvature. Some higher integer order curvature flows involving $P_{k}^{g}$, such as $Q$-curvature flow, have been studied in [12, 60, 4, 14, 47] and so on.

Making use of a generalized Dirichlet to Neumann map, Graham and Zworski [42] introduced a meromorphic family of conformally invariant operators on the conformal infinity 
of asymptotically hyperbolic manifolds (see Mazzeo and Melrose [61]). Recently, Chang and González [22] reconciled the way of Graham and Zworski to define conformally invariant operators $P_{\sigma}^{g}$ of non-integer order $\sigma \in\left(0, \frac{n}{2}\right)$ and the localization method of Caffarelli and Silvestre [19] for factional Laplacian $(-\Delta)^{\sigma}$ on the Euclidean space $\mathbb{R}^{n}$. These lead naturally to a fractional order curvature $R_{\sigma}^{g}:=P_{\sigma}^{g}(1)$, which will be called $\sigma$-curvature in this paper. There have been several work on these conformally invariant equations of fractional order and prescribing $\sigma$-curvatures problem (fractional Yamabe problem and fractional Nirenberg problem), see, e.g., [68], [39], [40], [51] and [52]. In this paper we study some flow of this fractional order curvature $R_{\sigma}^{g}$ associated with $P_{\sigma}^{g}$ on the standard conformal sphere $\left(\mathbb{S}^{n},\left[g_{\mathbb{S}^{n}}\right]\right)$, which is the conformal infinity of the Poincaré disk with the standard Poincaré metric.

Let $g$ be a representative in the conformal class $\left[g_{\mathbb{S}^{n}}\right]$ and write $g=v^{\frac{4}{n-2 \sigma}} g_{\mathbb{S}^{n}}$, where $v$ is positive and smooth on $\mathbb{S}^{n}$. Then we have

$$
P_{\sigma}^{g}(\phi)=v^{-\frac{n+2 \sigma}{n-2 \sigma}} P_{\sigma}^{g_{\mathbb{S} n}}(\phi v) \text { for any } \phi \in C^{\infty}\left(\mathbb{S}^{n}\right) .
$$

$P_{\sigma}^{g_{\S} n}$, which is simply written as $P_{\sigma}$, has the formula (see, e.g., [10])

$$
P_{\sigma}=\frac{\Gamma\left(B+\frac{1}{2}+\sigma\right)}{\Gamma\left(B+\frac{1}{2}-\sigma\right)}, \quad B=\sqrt{-\Delta_{g_{\Phi^{n}}}+\left(\frac{n-1}{2}\right)^{2}},
$$

where $\Gamma$ is the Gamma function and $\Delta_{g_{\mathbb{S}}}$ is the Laplace-Beltrami operator on $\left(\mathbb{S}^{n}, g_{\mathbb{S}^{n}}\right)$. Let $Y^{(k)}$ be a spherical harmonic of degree $k \geq 0$. Since $-\Delta_{g_{\mathbb{S}}} Y^{(k)}=k(k+n-1) Y^{(k)}$,

$$
B\left(Y^{(k)}\right)=\left(k+\frac{n-1}{2}\right) Y^{(k)} \quad \text { and } \quad P_{\sigma}\left(Y^{(k)}\right)=\frac{\Gamma\left(k+\frac{n}{2}+\sigma\right)}{\Gamma\left(k+\frac{n}{2}-\sigma\right)} Y^{(k)} .
$$

It is also well-known (see, e.g., [65]) that $P_{\sigma}$ is the inverse of the spherical Riesz potential

$$
K^{\sigma}(f)(\xi)=c_{n, \sigma} \int_{\mathbb{S}^{n}} \frac{f(\zeta)}{|\xi-\zeta|^{n-2 \sigma}} \mathrm{d} v o l_{\mathbb{S}^{n}}(\zeta), \quad f \in L^{p}\left(\mathbb{S}^{n}\right)
$$

where $c_{n, \sigma}=\frac{\Gamma\left(\frac{n-2 \sigma}{2}\right)}{2^{2 \sigma} \pi^{n / 2} \Gamma(\sigma)}, 1 \leq p<\infty$ and $|\cdot|$ is the Euclidean distance in $\mathbb{R}^{n+1}$. The inverses of spherical Riesz potentials have been constructed in terms of singular integrals in [67] and [69]. When $\sigma \in(0,1)$, Pavlov and Samko [67] showed that if $v=K^{\sigma}(f)$ for some $f \in L^{p}\left(\mathbb{S}^{n}\right)$, then

$$
P_{\sigma}(v)(\xi)=P_{\sigma}(1) v(\xi)+c_{n,-\sigma} \int_{\mathbb{S}^{n}} \frac{v(\xi)-v(\zeta)}{|\xi-\zeta|^{n+2 \sigma}} \mathrm{d} v o l_{\mathbb{S}^{n}}(\zeta)
$$

where $c_{n,-\sigma}=\frac{2^{2 \sigma} \sigma \Gamma\left(\frac{n+2 \sigma}{2}\right)}{\pi^{\frac{n}{2}} \Gamma(1-\sigma)}$ and $\int_{\mathbb{S}^{n}}$ is understood as $\lim _{\varepsilon \rightarrow 0} \int_{|x-y|>\varepsilon}$ in $L^{p}\left(\mathbb{S}^{n}\right)$ sense. 
Consider the normalized total $\sigma$-curvature functional

$$
S(g)=\operatorname{vol}_{g}\left(\mathbb{S}^{n}\right)^{\frac{2 \sigma-n}{n}} \int_{\mathbb{S}^{n}} R_{\sigma}^{g} \mathrm{~d} v o l_{g}, \quad g \in\left[g_{\mathbb{S}^{n}}\right] .
$$

The negative gradient flow of $S$ takes the form

$$
\frac{\partial g}{\partial t}=-\frac{n-2 \sigma}{2 n}\left(\operatorname{vol}_{g}\left(\mathbb{S}^{n}\right)\right)^{\frac{2 \sigma-n}{n}}\left(R_{\sigma}^{g}-r_{\sigma}^{g}\right) g
$$

where $r_{\sigma}^{g}$ is the average of $R_{\sigma}^{g}$. It is easy to verify that this flow preserves the conformal class and the volume of $\mathbb{S}^{n}$. By a rescaling of the time variable, we obtain the following evolution equation

$$
\frac{\partial g}{\partial t}=-\left(R_{\sigma}^{g}-r_{\sigma}^{g}\right) g
$$

If we write $g(t)=v^{\frac{4}{n-2 \sigma}}(\cdot, t) g_{\mathbb{S}^{n}}$, then after rescaling the time variable, (7) can be written in an equivalent form

$$
\frac{\partial v^{N}}{\partial t}=-P_{\sigma}(v)+r_{\sigma}^{g} v^{N} \quad \text { on } \mathbb{S}^{n}
$$

where $N=(n+2 \sigma) /(n-2 \sigma)$.

Let $\mathcal{N}$ be the north pole of $\mathbb{S}^{n}$ and

$$
F: \mathbb{R}^{n} \rightarrow \mathbb{S}^{n} \backslash\{\mathcal{N}\}, \quad x \mapsto\left(\frac{2 x}{1+|x|^{2}}, \frac{|x|^{2}-1}{|x|^{2}+1}\right)
$$

be the inverse of stereographic projection from $\mathbb{S}^{n} \backslash\{\mathcal{N}\}$ to $\mathbb{R}^{n}$. Then

$$
\left(P_{\sigma}(\phi)\right) \circ F=\left|J_{F}\right|^{-\frac{n+2 \sigma}{2 n}}(-\Delta)^{\sigma}\left(\left|J_{F}\right|^{\frac{n-2 \sigma}{2 n}}(\phi \circ F)\right) \quad \text { for } \phi \in C^{\infty}\left(\mathbb{S}^{n}\right),
$$

where $\left|J_{F}\right|=\left(\frac{2}{1+|x|^{2}}\right)^{n}$ and $(-\Delta)^{\sigma}$ is the fractional Laplacian operator (see, e.g., [73]). Hence $u(x, t):=\left|J_{F}\right|^{\frac{n-2 \sigma}{2 n}} v(F(x), t)$ satisfies

$$
\frac{\partial u^{N}}{\partial t}=-(-\Delta)^{\sigma} u+r_{\sigma}^{g} u^{N} \quad \text { in } \mathbb{R}^{n}
$$

We will call (7), (8) or (10) as a (normalized) fractional Yamabe flow when $\sigma \in(0,1)$.

As observed in [22] that the operator $P_{1 / 2}^{g}$ is related to the Yamabe problem on manifolds with boundary (see, e.g., [24, 33, 34, 45, 46]), this fractional Yamabe flow (7) with $\sigma=1 / 2$ is related to some generalization of Yamabe flow for manifolds with boundary studied in [11].

Throughout this paper we always assume that $0<\sigma<1$ without otherwise stated. Our first result is the long time existence and convergence of solutions of (7) for any initial data in the conformal class of $g_{\mathbb{S}^{n}}$. 
Theorem 1.1. Let $g(0) \in\left[g_{\mathbb{S}^{n}}\right]$ be a smooth metric on $\mathbb{S}^{n}$ for $n \geq 2$. Then the fractional Yamabe flow (7) with initial metric $g(0)$ exists for all time $0<t<\infty$. Furthermore, there exists a smooth metric $g_{\infty} \in\left[g_{\mathbb{S}^{n}}\right]$ such that

$$
R_{\sigma}^{g_{\infty}}=r_{\sigma}^{g_{\infty}} \text { and } \quad \lim _{t \rightarrow \infty}\left\|g(t)-g_{\infty}\right\|_{C^{l}\left(\mathbb{S}^{n}\right)}=0
$$

for all positive integers $l$.

Remark 1.2. If we write $g_{\infty}=v_{\infty}^{\frac{4}{n-2 \sigma}} g_{\mathbb{S}^{n}}$ where $v_{\infty}$ is a smooth and positive function on $\mathbb{S}^{n}$, then Theorem 1.1 implies that $v_{\infty}$ satisfies

$$
P_{\sigma}\left(v_{\infty}\right)=r_{\sigma}^{g_{\infty}} \cdot v_{\infty}^{\frac{n+2 \sigma}{n-2 \sigma}}
$$

whose solutions are classified in [23] and [57].

We also consider the unnormalized fractional Yamabe flow

$$
\frac{\partial v^{N}}{\partial t}=-P_{\sigma}(v) \quad \text { on } \mathbb{S}^{n} \times(0, \infty), \quad \text { or } \quad \frac{\partial u^{N}}{\partial t}=-(-\Delta)^{\sigma} u \quad \text { in } \mathbb{R}^{n} \times(0, \infty) \text {. }
$$

The second one is a fractional porous medium equation studied, e.g., in [2, 28, 20, 29, 53], where it is taken the form

$$
\left\{\begin{aligned}
u_{t} & =-(-\Delta)^{\sigma}\left(|u|^{m-1} u\right) & & \text { in } \mathbb{R}^{n} \times(0, \infty), \\
u(\cdot, 0) & =u_{0} & & \text { in } \mathbb{R}^{n}
\end{aligned}\right.
$$

with $m=\frac{n-2 \sigma}{n+2 \sigma}, \sigma \in(0,1)$. Models of this kind of fractional diffusion equations arise, e.g., in statistical mechanics [48, 49, 50] and heat control [2].

These fractional diffusion equations have been systematically studied in [28] and [29]. It is proved in [29] that if $u_{0} \in L^{1}\left(\mathbb{R}^{n}\right) \cap L^{p}\left(\mathbb{R}^{n}\right)$ for $p>4 n /(n+2 \sigma)$, then there exists a unique strong solution (see [29] for the definition) of (11), and the solution will extinct in finite time. More precisely, if $u_{0}$ is nonnegative but not identically equals to zero, then there exists a $T=T\left(u_{0}\right) \in(0, \infty)$ such that $u(x, t)>0$ in $\mathbb{R}^{n} \times(0, T)$, and $u(x, T) \equiv 0$ in $\mathbb{R}^{n}$.

We are interested in analyzing the exact behavior of solutions of (11) near the extinction time for fast decaying initial data. In the classical case $\sigma=1$, the extinction profiles of solutions of porous medium equations have been described in the results of [37, 30, 27, 8, 9] and so on.

Theorem 1.3 below describes the extinction profile of $u(x, t)$, which extends the result of del Pino and Saéz in [30] for $\sigma=1$ to $\sigma \in(0,1)$.

Theorem 1.3. Assume that $u_{0} \in C^{2}\left(\mathbb{R}^{n}\right)$ is positive in $\mathbb{R}^{n}$ for $n \geq 2$. In addition, we assume, for $\left(u_{0}^{m}\right)_{0,1}(x):=|x|^{2 \sigma-n} u_{0}^{m}\left(x /|x|^{2}\right)$, that $\left(u_{0}^{m}\right)_{0,1}(x)$ can be extended to a positive and $C^{2}$ 
function near the origin. There exist $\lambda>0$ and $x_{0} \in R^{n}$ such that if $T=T\left(u_{0}\right) \in(0, \infty)$ denotes the extinction time of the solution of (11), then

$$
(T-t)^{-1 /(1-m)} u(x, t)=k(n, \sigma)\left(\frac{\lambda}{\lambda^{2}+\left|x-x_{0}\right|^{2}}\right)^{\frac{n+2 \sigma}{2}}+\theta(x, t)
$$

with

$$
\sup _{\mathbb{R}^{n}}\left(1+|x|^{n+2 \sigma}\right) \theta(x, t) \rightarrow 0 \quad \text { as } t \rightarrow T,
$$

where $k(n, \sigma)=2^{\frac{n-2}{2}}\left((1-m) P_{\sigma}(1)\right)^{\frac{n-2 \sigma}{4 \sigma}}$ and $P_{\sigma}(1)$ is given in (4).

Some estimates of the extinction time $T$ involving the sharp constant in Sobolev inequalities are postponed to Lemma6.1 in Section 6

An application of Theorem 1.3 is an improvement of some Sobolev inequality. A sharp form of the standard Sobolev inequality in $\mathbb{R}^{n}(n \geq 3)$ asserts that

$$
S_{n}\|\nabla u\|_{L^{2}\left(\mathbb{R}^{n}\right)}-\|u\|_{L^{\frac{2 n}{n-2}\left(\mathbb{R}^{n}\right)}} \geq 0
$$

for all $u \in \dot{H}^{1}\left(\mathbb{R}^{n}\right)=\left\{u \in L^{\frac{2 n}{n-2}}\left(\mathbb{R}^{n}\right): \nabla u \in L^{2}\left(\mathbb{R}^{n}\right)\right\}$, where $S_{n}$ is the sharp constant obtained in [3] and [74].

There have been many results on remainder terms of Sobolev inequalities (see, e.g., [17. 16, 6, 26, 21, 32]), which give various lower bounds of the left-hand side of (12).

Recently, Carlen, Carrillo and Loss in [21] noticed that some Hardy-Littlewood-Sobolev inequalities in dimension $n \geq 3$ and some special Gagliardo-Nirenberg inequalities can be related by a fast diffusion equation. In another recent paper [32], Dolbeault used a fast diffusion flow to obtain an optimal integral remainder term which improves (12) in dimension $n \geq 5$. Inspired by [21] and [32], we consider some Sobolev inequality involving fractional Sobolev spaces of order $\sigma \in(0,1)$, compared to those mentioned above corresponding to $\sigma=1$.

For any $\sigma \in(0,1)$, the Sobolev inequality (see, e.g., [73] or [31]) asserts that

$$
\|u\|_{L^{2^{*}(\sigma)}}^{2} \leq S_{n, \sigma}\|u\|_{\dot{H}^{\sigma}}^{2}, \quad \forall u \in \dot{H}^{\sigma}\left(\mathbb{R}^{n}\right),
$$

where $2^{*}(\sigma)=\frac{2 n}{n-2 \sigma}, S_{n, \sigma}$ is the optimal constant and $\dot{H}^{\sigma}\left(\mathbb{R}^{n}\right)$ is the closure of $C_{c}^{\infty}\left(\mathbb{R}^{n}\right)$ under the norm

$$
\|u\|_{\dot{H}^{\sigma}}=\left\|(-\Delta)^{\sigma / 2} u\right\|_{L^{2}\left(\mathbb{R}^{n}\right)} .
$$

The optimal constant $S_{n, \sigma}$ in the Sobolev inequality (13) is obtained by Lieb [58] and is achieved by $u(x)=\left(1+|x|^{2}\right)^{-\frac{n-2 \sigma}{2}}$. The Hardy-Littlewood-Sobolev inequality

$$
S_{n, \sigma}\|u\|_{L^{\frac{2 n}{n+2 \sigma}}}^{2} \geq \int_{\mathbb{R}^{n}} u(-\Delta)^{-\sigma} u \mathrm{~d} x, \quad \forall u \in L^{\frac{2 n}{n+2 \sigma}}\left(\mathbb{R}^{n}\right)
$$


involves the same optimal constant $S_{n, \sigma}$, where $(-\Delta)^{-\sigma}$ is a Riesz potential defined by

$$
(-\Delta)^{-\sigma} u(x)=c_{n, \sigma} \int_{\mathbb{R}^{n}} \frac{u(y)}{|x-y|^{n-2 \sigma}} \mathrm{d} y .
$$

We investigate the relation between (13) and (15) via the fractional diffusion equation (11), i.e.

$$
u_{t}=-(-\Delta)^{\sigma} u^{m}
$$

with $m=1 / N=\frac{n-2 \sigma}{n+2 \sigma}$. If we suppose that the initial data $u_{0}$ satisfies the assumptions in Theorem 1.3, then by Theorem 5.1 (which is used to prove Theorem 1.3) $u(\cdot, t)$ is positive and smooth in $\mathbb{R}^{n}$ before its extinction time, and for any fixed $t, u(x, t)=O\left(|x|^{-n-2 \sigma}\right)$ as $x \rightarrow \infty$. We define

$$
H(t):=H_{n, \sigma}(u(\cdot, t))=\int_{\mathbb{R}^{n}} u(-\Delta)^{-\sigma} u \mathrm{~d} x-S_{n, \sigma}\|u\|_{L^{\frac{2 n}{n+2 \sigma}}}^{2} .
$$

It follows from direct computations that $\frac{\mathrm{d}}{\mathrm{d} t} H \geq 0$ (see Proposition 6.1).

Consequently, one can prove (15), which is equivalent to $H \leq 0$, by showing

$$
\limsup _{t \rightarrow T} H(t) \leq 0
$$

where $T$ is the extinction time of (11). This can be seen clearly from Theorem 5.1 From this and Proposition 6.1 we also recover that $u^{m}$ is an extremal of (13) if $u$ is an extremal of (15).

Along this fractional fast diffusion flow, we can improve the Sobolev inequality (13), via a quantitative estimate of the remainder term. This improvement also holds as $\sigma \rightarrow 1$ and it extends some work of Dolbeault in [32].

Theorem 1.4. Assume that $\sigma \in(0,1)$ and $n>4 \sigma$. There exists a positive constant $C$ depending only on $n$ and $\sigma$ such that for any nonnegative function $u \in \dot{H}^{\sigma}\left(\mathbb{R}^{n}\right)$ we have

$$
\begin{aligned}
S_{n, \sigma}\left\|u^{N}\right\|_{L^{\frac{2 n}{n+2 \sigma}}}^{2} & -\int_{\mathbb{R}^{n}} u^{N}(-\Delta)^{-\sigma} u^{N} \mathrm{~d} x \\
& \leq C\|u\|_{L^{2^{*}(\sigma)}}^{\frac{8 \sigma}{n-2 \sigma}}\left(S_{n, \sigma}\|u\|_{\dot{H}^{\sigma}}^{2}-\|u\|_{L^{2^{*}(\sigma)}}^{2}\right),
\end{aligned}
$$

where $N=\frac{n+2 \sigma}{n-2 \sigma}$. Moreover, $C$ can be taken as $\frac{n+2 \sigma}{n}\left(1-e^{-\frac{n}{2 \sigma}}\right) S_{n, \sigma}$.

The operators $P_{\sigma}$ and $(-\Delta)^{\sigma}$ are nonlocal, pseudo-differential operators. Generally speaking, strong maximum principles and Harnack inequalities might fail for nonlocal operators, see, e.g., a counterexample in [54]. The counterexample in [54] shows that the local non-negativity of solutions of certain nonlocal equations is not enough to guarantee local strong maximum principles and Harnack inequalities. However, if solutions are assumed to be globally nonnegative, then various strong maximum principles and Harnack inequalities have been obtained in, e.g., [18], [75] and [51]. 
In this paper, we establish a strong maximum principle and a Hopf lemma for odd solutions of some linear nonlocal parabolic equations, which should be of independent interest. Our proofs make use of the expression (19) of $(-\Delta)^{\sigma}$. The odd function in Lemma 2.4 will serve as a barrier function, which allows us to obtain a Hopf lemma.

The paper is organized as follows. In Section 2 we prove a strong maximum principle and a Hopf lemma for odd solutions of some linear nonlocal parabolic equations. These are two crucial ingredients in our arguments. In Section 3 we prove a Harnack inequality via the method of moving planes. This idea is essentially due to R. Ye [76]. In Section 4] we show Schauder estimates, existence and convergence of solutions of the fractional Yamabe flows. Section 5 is devoted to proving Theorem 1.3 We rewrite (11) on $\mathbb{S}^{n}$ and apply the methods in Section 4. The improvement of the Sobolev inequality, Theorem 1.4 is proved in Section 6. Our proofs of Theorem 1.3 and Theorem 1.4 adapt some arguments in [30] and [32], respectively. In Appendix A we provide an analog of L. Simon's uniqueness theorem (see [72]) for negative gradient flows in our nonlocal flow setting. In Appendix B we present some interpolation inequalities and elementary computations which are used in Section 4

Acknowledgements: Both authors thank Prof. Y.Y. Li for encouragements and useful discussions. We also thank Prof. N. Sesum for bringing paper [30] to our attention. Tianling Jin was partially supported by a University and Louis Bevier Dissertation Fellowship at Rutgers University and Rutgers University School of Art and Science Excellence Fellowship. Jingang Xiong was partially supported by CSC project for visiting Rutgers University and NSFC No. 11071020. He is very grateful to the Department of Mathematics at Rutgers University for the kind hospitality.

Added in April 2012: After the first author gave a seminar at Princeton University in April 2012, Professor Rupert L. Frank informed us that they studied strong maximum principles and Hopf lemma for odd solutions for nonlocal elliptic equations in [36]. This also leads us to another reference [7] where some extension of Hopf lemma to non-local contexts is proved. Our case and the proofs are different from theirs. We thank Professor Rupert L. Frank for his interests in our work and bringing the references [7, 36] to our attention.

\section{A strong maximum principle and a Hopf lemma for nonlocal parabolic equations}

Let $x=\left(x^{\prime}, x_{n}\right) \in \mathbb{R}^{n}, \mathbb{R}_{+}^{n}=\left\{x: x_{n}>0, x \in \mathbb{R}^{n}\right\}$. Recall (see, e.g., [71]) that for $\sigma \in(0,1)$, if $u$ is bounded in $\mathbb{R}^{n}$ and $C^{2}$ near $x$, then $(-\Delta)^{\sigma} u$ is continuous near $x$, and

$$
(-\Delta)^{\sigma} u(x)=c_{n,-\sigma} \text { P.V. } \int_{\mathbb{R}^{n}} \frac{u(x)-u(y)}{|x-y|^{n+2 \sigma}} \mathrm{d} y .
$$


Here "P.V." means the principal value and $c_{n,-\sigma}$ is the constant in (6).

For simplicity, throughout the paper we denote $-(-\Delta)^{\sigma}$ as $\Delta^{\sigma}$ and will not keep writing the constant $c_{n,-\sigma}$ and "P.V." if there is no confusion.

Lemma 2.1. Let $w(x, t) \in C^{2,1}\left(\mathbb{R}^{n} \times \mathbb{R}\right)$ and $w(\cdot, t)$ be bounded in $\mathbb{R}^{n}$ for any fixed $t$. Suppose $w(x, t)$ satisfies $w\left(x^{\prime},-x_{n}, t\right)=-w\left(x^{\prime}, x_{n}, t\right)$ for all $(x, t)$ and

$$
\liminf _{x_{n} \geq 0,|x| \rightarrow \infty} w(x, t) \geq 0 \quad \text { for any fixed } t .
$$

If $w$ satisfies

$$
w_{t} \geq a(x, t) \Delta^{\sigma} w+b(x, t) w, \quad(x, t) \in \mathbb{R}_{+}^{n} \times(0, T],
$$

where $a(x, t)$ is continuous and positive in $\overline{\mathbb{R}_{+}^{n}} \times[0, T], b(x, t)$ is continuous and bounded in $\mathbb{R}_{+}^{n} \times[0, T]$, and $w(x, 0) \geq 0$ for all $x \in \mathbb{R}_{+}^{n}$, then $w(x, t) \geq 0$ in $\mathbb{R}_{+}^{n} \times[0, T]$.

Proof. Without loss of generality, we may assume $b(x, t) \leq 0$. Indeed, if we let

$$
\tilde{w}(x, t)=e^{-C t} w(x, t)
$$

for some constant $C$, then

$$
\tilde{w}_{t}=a(x, t) \Delta^{\sigma} \tilde{w}+(b(x, t)-C) \tilde{w} .
$$

Since $b$ is bounded, we can choose $C$ sufficiently large such that $b(x, t)-C \leq 0$ in $\mathbb{R}_{+}^{n} \times(0, T]$, and we only need to show that $\tilde{w}(x, t) \geq 0$ for all $(x, t) \in \mathbb{R}_{+}^{n} \times(0, T]$.

Suppose the contrary that there exists a point $\left(x_{0}, t_{0}\right) \in \mathbb{R}_{+}^{n} \times(0, T]$ such that

$$
0>w\left(x_{0}, t_{0}\right) \text {. }
$$

By the assumptions on $w$, we may assume $w\left(x_{0}, t_{0}\right)=\min _{\overline{\mathbb{R}}_{+}^{n} \times(0, T]} w$. It follows that

$$
w_{t}\left(x_{0}, t_{0}\right) \leq 0, \quad b\left(x_{0}, t_{0}\right) w\left(x_{0}, t_{0}\right) \geq 0 .
$$

It is clear that

$$
\begin{aligned}
\Delta^{\sigma} w\left(x_{0}, t_{0}\right) & =\int_{\mathbb{R}^{n}} \frac{w\left(y, t_{0}\right)-w\left(x_{0}, t_{0}\right)}{\left|x_{0}-y\right|^{n+2 \sigma}} \mathrm{d} y \\
& =\int_{\mathbb{R}_{+}^{n}} \frac{w\left(y, t_{0}\right)-w\left(x_{0}, t_{0}\right)}{\left|x_{0}-y\right|^{n+2 \sigma}} \mathrm{d} y+\int_{\mathbb{R}^{n} \backslash \mathbb{R}_{+}^{n}} \frac{w\left(y, t_{0}\right)-w\left(x_{0}, t_{0}\right)}{\left|x_{0}-y\right|^{n+2 \sigma}} \mathrm{d} y .
\end{aligned}
$$


By the change of variables $y=\left(z^{\prime},-z_{n}\right)$, we obtain

$$
\begin{aligned}
& \int_{\mathbb{R}^{n} \backslash \overline{\mathbb{R}_{+}^{n}}} \frac{w\left(y, t_{0}\right)-w\left(x_{0}, t_{0}\right)}{\left|x_{0}-y\right|^{n+2 \sigma}} \mathrm{d} y \\
& =\int_{\mathbb{R}_{+}^{n}} \frac{w\left(z^{\prime},-z_{n}, t_{0}\right)-w\left(x_{0}, t_{0}\right)}{\left|x_{0}-\left(z^{\prime},-z_{n}\right)\right|^{n+2 \sigma}} \mathrm{d} z \\
& =-\int_{\mathbb{R}_{+}^{n}} \frac{w\left(z^{\prime}, z_{n}, t_{0}\right)-w\left(x_{0}, t_{0}\right)}{\left|x_{0}-\left(z^{\prime},-z_{n}\right)\right|^{n+2 \sigma}} \mathrm{d} z-2 w\left(x_{0}, t_{0}\right) \int_{\mathbb{R}_{+}^{n}} \frac{1}{\left|x_{0}-\left(z^{\prime},-z_{n}\right)\right|^{n+2 \sigma}} \mathrm{d} z \\
& >-\int_{\mathbb{R}_{+}^{n}} \frac{w\left(z^{\prime}, z_{n}, t_{0}\right)-w\left(x_{0}, t_{0}\right)}{\left|x_{0}-\left(z^{\prime},-z_{n}\right)\right|^{n+2 \sigma}} \mathrm{d} z .
\end{aligned}
$$

where we used $w\left(z^{\prime},-z_{n}, t_{0}\right)=-w\left(z^{\prime}, z_{n}, t_{0}\right)$ and $w\left(x_{0}, t_{0}\right)<0$. Since $\left(x_{0}, t_{0}\right)$ is a minimum point of $w$ in $\overline{\mathbb{R}_{+}^{n}} \times(0, T]$, the simple inequality

$$
\frac{1}{\left|x_{0}-z\right|^{n+2 \sigma}}>\frac{1}{\left|x_{0}-\left(z^{\prime},-z_{n}\right)\right|^{n+2 \sigma}}, \quad \forall x_{0}, z \in \mathbb{R}_{+}^{n}
$$

yields that

$$
\Delta^{\sigma} w\left(x_{0}, t_{0}\right)>0
$$

Combining (21) and (22), we have a contradiction to (20).

Lemma 2.2. Let $w(x, t)$ be as in Lemma 2.1 Then for any fixed $t \in(0, T]$, we have $w(x, t)>$ 0 or $w(x, t) \equiv 0$ in $\mathbb{R}_{+}^{n}$.

Proof. As in the proof of Lemma 2.1, we may assume $b \leq 0$. Suppose that at $w\left(x_{0}, t_{0}\right)=0$ for some $\left(x_{0}, t_{0}\right) \in \mathbb{R}_{+}^{n} \times(0, T]$. From the proof of Lemma 2.1 we see that

$$
\Delta^{\sigma} w\left(x_{0}, t_{0}\right) \geq 0
$$

and equality holds if and only if $w\left(x, t_{0}\right)=w\left(x_{0}, t_{0}\right)$ for all $x \in \mathbb{R}_{+}^{n}$. Therefore, the lemma follows immediately from a simple contradiction argument.

Lemma 2.3. Let $w(x, t)$ be as in Lemma 2.1 Suppose $w\left(x_{0}, 0\right)>0$ for some $x_{0} \in \mathbb{R}_{+}^{n}$, then for any fixed $t \in(0, T]$, we have $w(x, t)>0$ in $\mathbb{R}_{+}^{n}$.

Proof. The proof follows from that of the parabolic strong maximum principle in [66], with suitable modifications for nonlocal equations. As before, we assume $b \leq 0$. Suppose that for some $t_{1}>0, w\left(\cdot, t_{1}\right)$ is zero at some point. It follows from Lemma 2.2 that $w\left(x, t_{1}\right) \equiv 0$. By the assumption on $w(\cdot, 0)$ and Lemma 2.2, we may assume that $w(x, t)>0$ for all $(x, t) \in$ $\mathbb{R}_{+}^{n} \times\left(t_{2}, t_{1}\right)$ for some $t_{2}>0$. 
Let $h(x, t)=\left(t_{1}-t_{*}\right)^{2}-\left|x-e_{n}\right|^{2}-\left(t-t_{*}\right)^{2}$ if $0 \leq x_{n} \leq 2$; and $h=0$ if $x_{n}>2$, where $e_{n}=\left(0^{\prime}, 1\right)$ and $t_{*}$ will be fixed later. Set

$$
H(x, t)= \begin{cases}h(x, t), & x \in \overline{\mathbb{R}_{+}^{n}} \\ -h\left(x^{\prime},-x_{n}, t\right), & x \in \mathbb{R}^{n} \backslash \overline{\mathbb{R}_{+}^{n}}\end{cases}
$$

Let $\bar{t} \in\left(t_{2}, t_{1}\right)$ be such that $\left(t_{1}-t_{*}\right)^{2}-\left(t-t_{*}\right)^{2} \leq \frac{1}{4}$ holds for $t \geq \bar{t}$. It is easy to see that there exists a positive constant $C_{1}$ independent of $t^{*}$ such that for any $(x, t) \in B_{1 / 2}\left(e_{n}\right) \times\left[\bar{t}, t_{1}\right]$,

$$
(-\Delta)^{\sigma} H(x, t) \leq C_{1}
$$

Thus, we can choose $t_{*}$ so negative that for any $(x, t) \in B_{1 / 2}\left(e_{n}\right) \times\left[\bar{t}, t_{1}\right]$,

$$
H_{t}(x, t)=2\left(t_{*}-t\right) \leq 2\left(t_{*}-t_{2}\right)<a(x, t) \Delta^{\sigma} H(x, t)+b(x, t) H(x, t) .
$$

Let $\varepsilon>0$ be a sufficiently small constant such that $w(x, \bar{t}) \geq \varepsilon H(x, \bar{t})$ for all $x \in \mathbb{R}_{+}^{n}$. We claim that $w(x, t) \geq \varepsilon H(x, t)$ in $\mathbb{R}_{+}^{n} \times\left(\bar{t}, t_{1}\right)$.

If not, then the (negative) minimal value of $\bar{w}:=w-\varepsilon H$ in $\mathbb{R}_{+}^{n} \times\left(\bar{t}, t_{1}\right)$ must be achieved in $B_{1 / 2}\left(e_{n}\right) \times\left(\bar{t}, t_{1}\right)$, say at $\left(x_{0}, t_{0}\right)$. Note that $\bar{w}\left(x^{\prime},-x_{n}, t\right)=-\bar{w}\left(x^{\prime}, x_{n}, t\right)$. Hence, by exactly the same argument in the proof of Lemma 2.1

$$
\partial_{t} \bar{w}\left(x_{0}, t_{0}\right) \leq 0, \quad \Delta^{\sigma} \bar{w}\left(x_{0}, t_{0}\right)>0 .
$$

Together with (23) and $b \leq 0$, we conclude that

$$
w_{t}\left(x_{0}, t_{0}\right)<a\left(x_{0}, t_{0}\right) \Delta^{\sigma} w\left(x_{0}, t_{0}\right)+b\left(x_{0}, t_{0}\right) w,
$$

which contradicts (20).

Hence, it follows from the above claim that $w_{t}\left(t_{1}, e_{n}\right) \leq-2 \varepsilon\left(t_{1}-t_{*}\right)<0$. But $w\left(x, t_{1}\right) \equiv$ 0 . These contradict (20).

Lemma 2.4. Let

$$
h(x)= \begin{cases}x_{n}\left(1-\left|x^{\prime}\right|^{2}\right), & \left|x_{n}\right|<1,\left|x^{\prime}\right|<1, \\ 0, & \text { otherwise. }\end{cases}
$$

Then there exists a positive constant $c_{0}$ depending only $n, \sigma$ such that

$$
\Delta^{\sigma} h(x) \geq-c_{0} x_{n}
$$

for all $x=\left(x^{\prime}, x_{n}\right)$ with $\left|x^{\prime}\right|<1,0 \leq x_{n}<1 / 8$. 
Proof. The lemma follows from rather involved calculations. By rotating the first $(n-1)$ coordinates, we only need to show (24) at point $a=\left(a_{1}, 0, \cdots, 0, a_{n}\right)$ with $0 \leq a_{1}<1$, $0 \leq a_{n}<1 / 8$.

Denote $B^{\prime}\left(x^{\prime}, R\right) \subset \mathbb{R}^{n-1}$ be the ball centered at $x^{\prime}$ with radius $R, \Omega=B^{\prime}(0,1) \times$ $(-1,1)$. In the following $C$ will be denoted as various positive constants which depend only on dimension $n$ and $\sigma$.

It follows from (19) that

$$
\begin{aligned}
\Delta^{\sigma} h(a) & =\int_{\mathbb{R}^{n}} \frac{h(x)-h(a)}{|x-a|^{n+2 \sigma}} \mathrm{d} x \\
& =\int_{\Omega} \frac{x_{n}\left(1-\left|x^{\prime}\right|^{2}\right)-a_{n}\left(1-\left|a^{\prime}\right|^{2}\right)}{|x-a|^{n+2 \sigma}} \mathrm{d} x-\int_{\Omega^{c}} \frac{a_{n}\left(1-\left|a^{\prime}\right|^{2}\right)}{|x-a|^{n+2 \sigma}} \mathrm{d} x \\
& =: I-a_{n} I I .
\end{aligned}
$$

Since $x_{n}\left(1-\left|x^{\prime}\right|^{2}\right)-a_{n}\left(1-\left|a^{\prime}\right|^{2}\right)=\left(x_{n}-a_{n}\right)\left(1-\left|x^{\prime}\right|^{2}\right)+a_{n}\left(\left|a^{\prime}\right|^{2}-\left|x^{\prime}\right|^{2}\right)$, we divide the integral $I$ into

$$
I_{1}:=\int_{\Omega} \frac{\left(x_{n}-a_{n}\right)\left(1-\left|x^{\prime}\right|^{2}\right)}{|x-a|^{n+2 \sigma}} \mathrm{d} x
$$

and

$$
a_{n} I_{2}:=a_{n} \int_{\Omega} \frac{\left(\left|a^{\prime}\right|^{2}-\left|x^{\prime}\right|^{2}\right)}{|x-a|^{n+2 \sigma}} \mathrm{d} x
$$

By symmetry and that $0 \leq a_{n}<1 / 8$,

$$
\begin{aligned}
I_{1} & =\int_{-1}^{-1+2 a_{n}} \int_{\left|x^{\prime}\right|<1} \frac{\left(x_{n}-a_{n}\right)\left(1-\left|x^{\prime}\right|^{2}\right)}{|x-a|^{n+2 \sigma}} \mathrm{d} x^{\prime} \mathrm{d} x_{n} \\
& \geq-C a_{n} .
\end{aligned}
$$

Using $\left|a^{\prime}\right|^{2}-\left|x^{\prime}\right|^{2}=-\left|x^{\prime}-a^{\prime}\right|^{2}+2 a^{\prime}\left(a^{\prime}-x^{\prime}\right)$, we write

$$
\begin{aligned}
I_{2} & =\int_{\Omega} \frac{-\left|x^{\prime}-a^{\prime}\right|^{2}}{|x-a|^{n+2 \sigma}} \mathrm{d} x+\int_{\Omega} \frac{2 a^{\prime} \cdot\left(a^{\prime}-x^{\prime}\right)}{|x-a|^{n+2 \sigma}} \mathrm{d} x \\
& =: I_{3}+I_{4} .
\end{aligned}
$$


Direct computations give

$$
\begin{aligned}
I_{3} & \geq-\int_{-2+a_{n}}^{2+a_{n}} \mathrm{~d} x_{n} \int_{\left|x^{\prime}-a^{\prime}\right|<2} \frac{-\left|x^{\prime}-a^{\prime}\right|^{2}}{|x-a|^{n+2 \sigma}} \mathrm{d} x^{\prime} \\
& =-2 \lim _{b \rightarrow 0^{+}} \int_{b}^{2} \mathrm{~d} y \int_{0}^{2} \frac{r^{2} r^{n-2}}{\left(r^{2}+y^{2}\right)^{\frac{n+2 \sigma}{2}}} \mathrm{~d} r \\
& =-2 \lim _{b \rightarrow 0^{+}} \int_{b}^{2} y^{1-2 \sigma} \mathrm{d} y \int_{0}^{2 / y} \frac{r^{n}}{\left(1+r^{2}\right)^{\frac{n+2 \sigma}{2}}} \mathrm{~d} r \\
& =-2 \lim _{b \rightarrow 0^{+}} \int_{b}^{2} y^{1-2 \sigma} \mathrm{d} y\left(\int_{1}^{2 / y} \frac{r^{n}}{\left(1+r^{2}\right)^{\frac{n+2 \sigma}{2}}} \mathrm{~d} r+\int_{0}^{1} \frac{r^{n}}{\left(1+r^{2}\right)^{\frac{n+2 \sigma}{2}}} \mathrm{~d} r\right) \\
& \geq-2 \lim _{b \rightarrow 0^{+}} \int_{b}^{2} y^{1-2 \sigma} \mathrm{d} y\left(\int_{1}^{2 / y} r^{-2 \sigma} \mathrm{d} r+1\right) \\
& \geq-C .
\end{aligned}
$$

Next, we are going to show

$$
I_{4}-I I \geq-C
$$

Let $D_{0}=\left(B^{\prime}(0,1) \cap B^{\prime}\left(2 a^{\prime}, 1\right)\right)$. Since $a^{\prime}=\left(a_{1}, 0, \cdots, 0\right)$, it follows from symmetry that

$$
\int_{D_{0} \times(-1,1)} \frac{2 a^{\prime} \cdot\left(a^{\prime}-x^{\prime}\right)}{|x-a|^{n+2 \sigma}} \mathrm{d} x^{\prime} \mathrm{d} x_{n}=0 .
$$

Thus,

$$
I_{4}=\int_{\left(B^{\prime}(0,1) \backslash D_{0}\right) \times(-1,1)} \frac{2 a^{\prime} \cdot\left(a^{\prime}-x^{\prime}\right)}{|x-a|^{n+2 \sigma}}>0 .
$$

Now we have two cases:

Case 1. if $\left|a^{\prime}\right| \leq \frac{\sqrt{2}}{2}$, then it is easy to see that $I I<C$ (the denominator is uniformly bounded). Hence, (28) holds.

Case 2. Suppose $\left|a^{\prime}\right|>\frac{\sqrt{2}}{2}$. We have

$$
\begin{aligned}
I I & =\int_{\Omega^{c} \cap\left(B^{\prime}\left(a^{\prime},\left|a^{\prime}\right|\right) \times(-1,1)\right)} \frac{1-\left|a^{\prime}\right|^{2}}{|x-a|^{n+2 \sigma}}+\int_{\Omega^{c} \backslash\left(B^{\prime}\left(a^{\prime},\left|a^{\prime}\right|\right) \times(-1,1)\right)} \frac{1-\left|a^{\prime}\right|^{2}}{|x-a|^{n+2 \sigma}} \\
& \leq \int_{\Omega^{c} \cap\left(B^{\prime}\left(a^{\prime},\left|a^{\prime}\right|\right) \times(-1,1)\right)} \frac{\left(1-\left|a^{\prime}\right|^{2}\right)}{|x-a|^{n+2 \sigma}}+C \\
& =: I I_{1}+C .
\end{aligned}
$$

Denote $D_{1}=\left(B^{\prime}\left(a^{\prime}, \sqrt{1-\left|a^{\prime}\right|^{2}}\right) \cap\left\{x_{1}<a_{1}\right\}\right) \backslash D_{0}$, and $D_{2}=\left(B^{\prime}\left(a^{\prime}, \sqrt{1-\left|a^{\prime}\right|^{2}}\right) \cap\right.$ $\left.\left\{x_{1}>a_{1}\right\}\right) \backslash D_{0}$. 
Note that for $x \in D_{1}$, we have $2\left|a^{\prime}\right|\left(\left|a^{\prime}\right|-x_{1}\right) \geq 1-\left|a^{\prime}\right|^{2}-\left|x^{\prime}-a^{\prime}\right|^{2}$. Therefore,

$$
\int_{D_{1} \times(-1,1)} \frac{2 a^{\prime} \cdot\left(a^{\prime}-x^{\prime}\right)}{|x-a|^{n+2 \sigma}}-\int_{\left.D_{2} \times(-1,1)\right)} \frac{1-\left|a^{\prime}\right|^{2}}{|x-a|^{n+2 \sigma}} \geq \int_{D_{1} \times(-1,1)} \frac{-\left|x^{\prime}-a^{\prime}\right|^{2}}{|x-a|^{n+2 \sigma}} .
$$

Observe that there exists a positive integer $m$, which depends only on $n$ and $\sigma$, such that

$$
\begin{aligned}
& m \int_{\left(B^{\prime}\left(0^{\prime}, 1\right) \backslash B^{\prime}\left(a^{\prime}, \sqrt{1-\left|a^{\prime}\right|^{2}}\right)\right) \times(-1,1)} \frac{1-\left|a^{\prime}\right|^{2}}{|x-a|^{n+2 \sigma}} \\
& \geq \int_{\left(B\left(a^{\prime},\left|a^{\prime}\right|\right) \backslash\left(B^{\prime}\left(0^{\prime}, 1\right) \cup B^{\prime}\left(a^{\prime}, \sqrt{1-\left|a^{\prime}\right|^{2}}\right)\right)\right) \times(-1,1)} \frac{1-\left|a^{\prime}\right|^{2}}{|x-a|^{n+2 \sigma}} .
\end{aligned}
$$

Also notice that for any $x \in B^{\prime}\left(0^{\prime}, 1\right) \backslash B^{\prime}\left(a^{\prime}, \sqrt{1-\left|a^{\prime}\right|^{2}}\right)$, we have

$$
0 \geq m\left(1-\left|a^{\prime}\right|^{2}-\left|x^{\prime}-a^{\prime}\right|^{2}\right) \text {. }
$$

Hence,

$$
\begin{aligned}
& m \int_{\left(B^{\prime}\left(0^{\prime}, 1\right) \backslash B^{\prime}\left(a^{\prime}, \sqrt{1-\left|a^{\prime}\right|^{2}}\right)\right) \times(-1,1)} \frac{\left|x^{\prime}-a^{\prime}\right|^{2}}{|x-a|^{n+2 \sigma}} \\
& \geq \int_{\left(B\left(a^{\prime},\left|a^{\prime}\right|\right) \backslash\left(B^{\prime}\left(0^{\prime}, 1\right) \cup B^{\prime}\left(a^{\prime}, \sqrt{1-\left|a^{\prime}\right|^{2}}\right)\right)\right) \times(-1,1)} \frac{1-\left|a^{\prime}\right|^{2}}{|x-a|^{n+2 \sigma}} .
\end{aligned}
$$

It follows that

$$
\begin{aligned}
I_{4} & -I I \\
\geq & -C+\int_{D_{1} \times(-1,1)} \frac{2 a^{\prime} \cdot\left(a^{\prime}-x^{\prime}\right)}{|x-a|^{n+2 \sigma}}-\int_{D_{2} \times(-1,1)} \frac{1-\left|a^{\prime}\right|^{2}}{|x-a|^{n+2 \sigma}} \\
& \left.\left.-\int_{\left(B ( a ^ { \prime } , | a ^ { \prime } | ) \backslash \left(B^{\prime}\left(0^{\prime}, 1\right) \cup B^{\prime}\left(a^{\prime}, \sqrt{1-\left|a^{\prime}\right|^{2}}\right)\right.\right.}\right)\right) \times(-1,1) \\
\geq & -C-m \int_{\left(B^{\prime}\left(0^{\prime}, 1\right) \backslash B^{\prime}\left(a^{\prime}, \sqrt{1-\left|a^{\prime}\right|^{2}}\right)\right) \times(-1,1)} \frac{1-\mid a^{\prime}}{|x-a|^{n+2 \sigma}} \\
\geq & -C-(m+1) I_{3} \\
\geq & -C .
\end{aligned}
$$

Therefore, (28) holds.

Finally, Lemma 2.4 follows from (25), (26), (27) and (28). 
Lemma 2.5. Let $w(x, t)$ be as in Lemma 2.1] Suppose $w\left(x_{0}, 0\right)>0$ for some $x_{0} \in \mathbb{R}_{+}^{n}$, then for any fixed $t \in(0, T]$, we have $\partial_{x_{n}} w\left(x^{\prime}, 0, t\right)>0$.

Proof. Let

$$
g(x)= \begin{cases}-1, & \text { in } B^{\prime}(0,1) \times(-2,-1) \\ 1, & \text { in } B^{\prime}(0,1) \times(1,2) \\ 0, & \text { otherwise }\end{cases}
$$

where $B^{\prime}(0,1)$ denotes the $n-1$ dimensional unit ball centered at 0 . For any $x \in B^{\prime}(0,1) \times$ $(0,1 / 8)$, we have

$$
\begin{aligned}
\Delta^{\sigma} g(x) & =\int_{\mathbb{R}^{n}} \frac{g(y)-g(x)}{|y-x|^{n+2 \sigma}} \mathrm{d} y \\
& =\int_{B^{\prime}(0,1) \times(1,2)} \frac{1}{|y-x|^{n+2 \sigma}} \mathrm{d} y-\int_{B^{\prime}(0,1) \times(-2,-1)} \frac{1}{|y-x|^{n+2 \sigma}} \mathrm{d} y \\
& =\int_{B^{\prime}(0,1) \times(1,2)} \frac{1}{\left|y-\left(x^{\prime}, x_{n}\right)\right|^{n+2 \sigma}}-\frac{1}{\left|y-\left(x^{\prime},-x_{n}\right)\right|^{n+2 \sigma}} \mathrm{d} y \\
& =\int_{B^{\prime}(0,1) \times(1,2)} \int_{0}^{1}-\frac{\mathrm{d}}{\mathrm{d} s}\left(\frac{1}{\left|y-x+2 s x_{n} e_{n}\right|^{n+2 \sigma}}\right) \mathrm{d} s \mathrm{~d} y \\
& =(n+2 \sigma) \int_{B^{\prime}(0,1) \times(1,2)} \int_{0}^{1} \frac{4\left(y_{n}-x_{n}\right) x_{n}+8 s x_{n}^{2}}{\left|y-x+2 s x_{n} e_{n}\right|^{n+2+2 \sigma}} \mathrm{d} s \mathrm{~d} y \\
& \geq c_{1} x_{n},
\end{aligned}
$$

where $c_{1}>0$ depends only on $n$ and $\sigma$.

For any fixed $t_{0} \in(0, T]$, define

$$
H(x, t)=h(x)\left(\frac{t_{0}^{2}}{1+t_{0}^{2}}-\left(t-t_{0}\right)^{2}\right)+k g(x),
$$

where $h$ is as in Lemma2.4 We can choose a sufficiently large constant $k$ such that

$$
H_{t}(x, t) \leq a(x, t) \Delta^{\sigma} H+b(x, t) H(x, t),
$$

for all $x \in B^{\prime}(0,1) \times(0,1 / 8)$ and $t \in\left(t_{0}-t_{0} / \sqrt{1+t_{0}^{2}}, t_{0}\right]$.

It follows from Lemma 2.3 that $w(\cdot, t)>0$ in $\mathbb{R}_{+}^{n}$ for any fixed $t \in(0, T]$. Making a similar argument to the poof of Lemma 2.3, we can show that there exists a small positive constant $\varepsilon$ such that $w \geq \varepsilon H$ for all $t \in\left(0, t_{0}\right]$. Therefore, $\partial_{x_{n}} w\left(x^{\prime}, 0, t_{0}\right)>0$ and Lemma 2.5 follows immediately.

Now we apply the above strong maximum principle and Hopf lemma to fractional Yamabe flow equations. 
Suppose that $v$ is a positive smooth solution of $(8)$ in $\mathbb{S}^{n} \times[0, T]$. Hence

$$
u(x, t)=\left(\frac{2}{1+|x|^{2}}\right)^{\frac{n-2 \sigma}{2}} v(F(x), t)
$$

satisfies (10). For a given real number $\lambda$, define

$$
\Sigma_{\lambda}=\left\{x=\left(x^{\prime}, x_{n}\right): x_{n} \geq \lambda\right\},
$$

and let $x^{\lambda}=\left(x^{\prime}, 2 \lambda-x_{n}\right)$ and $u_{\lambda}(x, t)=u\left(x^{\lambda}, t\right)$. It is clear that $u_{\lambda}$ also satisfies (10).

Proposition 2.1. Suppose that $u(x, 0)-u_{\lambda}(x, 0) \geq 0$ in $\Sigma_{\lambda}$, then for any fixed $t \in(0, T]$, we have $u(x, t)-u_{\lambda}(x, t) \geq 0$ in $\Sigma_{\lambda}$.

Proof. Let $w(x, t)=u(x, t)-u_{\lambda}(x, t)$. Then $w$ satisfies

$$
w_{t}=a(x, t) \Delta^{\sigma} w+b(x, t) w
$$

where $a(x, t)=\frac{1}{N u^{N-1}}$ and $b(x, t)=\frac{(1-N)(-\Delta)^{\sigma} u_{\lambda}}{N} \int_{0}^{1} \frac{1}{\left(\tau u+(1-\tau) u_{\lambda}\right)^{N}} d \tau+\frac{r_{\sigma}^{g}}{N}$ is bounded. Note that $w\left(x^{\prime}, x_{n}+\lambda, t\right)$ satisfies all the conditions in Lemma 2.1] Thus Proposition 2.1 follows from Lemma 2.1

Proposition 2.2. Assume the conditions in Proposition 2.1 then for any fixed $t \in(0, T]$, we have $u(x, t)-u_{\lambda}(x, t)>0$ or $u(x, t)-u_{\lambda}(x, t) \equiv 0$ in $\Sigma_{\lambda}$.

Proof. It follows from Proposition 2.1 and Lemma2.2.

Proposition 2.3. Assume the conditions in Proposition 2.1] In addition, we suppose that $u\left(x_{0}, 0\right)-u_{\lambda}\left(x_{0}, 0\right)>0$ for some $x_{0} \in \Sigma_{\lambda}$, then for any fixed $t \in(0, T]$, we have $u(x, t)-$ $u_{\lambda}(x, t)>0$ in $\Sigma_{\lambda}$ and $\partial_{x_{n}} u\left(x^{\prime}, \lambda, t\right)>0$.

Proof. It follows from Proposition 2.1, Lemma 2.3 and Lemma 2.5

\section{Harnack inequality for a fractional Yamabe flow}

Based on the results proved in the previous section, we are going to establish the following Harnack inequality.

Theorem 3.1. Let $v$ be a $C^{3,1}$ positive function on $\mathbb{S}^{n} \times\left[0, T^{*}\right)$ and satisfy

$$
\frac{\partial v^{N}}{\partial t}=-P_{\sigma}(v)+b(t) v^{N}, \quad \text { on } \mathbb{S}^{n} \times\left(0, T^{*}\right)
$$


where $b(t) \in C\left(\left[0, T^{*}\right)\right)$. Then there exists a positive constant $C>0$ depending only on $n, \sigma, \inf _{\mathbb{S}^{n}} v(\cdot, 0)$ and $\|v(\cdot, 0)\|_{C^{3}\left(\mathbb{S}^{n}\right)}$ such that

$$
\max _{\mathbb{S}^{n}} v(\cdot, t) \leq C \min _{\mathbb{S}^{n}} v(\cdot, t)
$$

for any fixed $t \in\left(0, T^{*}\right)$.

Proof. As mentioned in the introduction, the idea of this proof is essentially due to Ye [76]. We will show that

$$
\sup _{\mathbb{S}^{n}} \frac{\left|\nabla_{\mathbb{S}^{n}} v\right|}{|v|} \leq C \quad \text { for all } s \in\left(0, T^{*}\right) .
$$

Let $q_{0} \in \mathbb{S}^{n}$. Without loss of generality, we may assume that $q_{0}$ is the north pole. Consider the inverse of the stereographic projection from the north pole $F: \mathbb{R}^{n} \rightarrow \mathbb{S}^{n}$ :

$$
F\left(x_{1}, \cdots, x_{n}\right)=\left(\frac{2 x}{1+x^{2}}, \frac{x^{2}-1}{x^{2}+1}\right) .
$$

We also denote $G: \mathbb{R}^{n} \rightarrow \mathbb{S}^{n}$ as the inverse of the stereographic projection from the south pole, namely $G(x)=F\left(x /|x|^{2}\right)$. Let

$$
u(x, s)=\left(\frac{2}{1+|x|^{2}}\right)^{\frac{n-2 \sigma}{2}} v(F(x), s), \quad \bar{u}(x, s)=\left(\frac{2}{1+|x|^{2}}\right)^{\frac{n-2 \sigma}{2}} v(G(x), s) .
$$

Then $u, \bar{u} \in C^{3,1}\left(\mathbb{R}^{n} \times\left[0, T^{*}\right)\right)$ and both satisfy

$$
\frac{\partial u^{N}}{\partial t}=\Delta^{\sigma} u+b(t) u^{N}, \quad \text { on } \mathbb{R}^{n} \times\left[0, T^{*}\right)
$$

$u(\cdot, s)$ has a Taylor expansion "at infinity" of the form

$$
u(x, s)=\frac{2^{(n-2 \sigma) / 2}}{|x|^{n-2 \sigma}}\left(a_{0}+\frac{a_{i} x_{i}}{|x|^{2}}+\left(a_{i j}-\frac{n-2 \sigma}{2} \delta_{i j}\right) \frac{x_{i} x_{j}}{|x|^{4}}+O\left(|x|^{-3}\right)\right) .
$$

Similarly, the partial derivatives of $u(\cdot, t)$ have Taylor expansions "at infinity" of the form

$$
\begin{aligned}
\frac{\partial u}{\partial x_{i}}(x, s)= & 2^{\frac{n-2 \sigma}{2}}\left(-\frac{n-2 \sigma}{|x|^{n-2 \sigma+2}} x_{i}\left(a_{0}+\frac{a_{j} x_{j}}{|x|^{2}}\right)+\frac{a_{i}}{|x|^{n-2 \sigma+2}}-\frac{2 x_{i} a_{j} x_{j}}{|x|^{n-2 \sigma+4}}\right) \\
& +O\left(|x|^{-(n-2 \sigma+3)}\right) .
\end{aligned}
$$

Here

$$
\begin{aligned}
a_{0}(s) & =v\left(q_{0}, s\right), \\
a_{i}(s) & =\frac{\partial(v(\cdot, s) \circ G)}{\partial x_{i}}(0), \\
a_{i j}(s) & =\frac{\partial^{2}(v(\cdot, s) \circ G)}{2 \partial x_{i} x_{j}}(0) .
\end{aligned}
$$


Let $y_{i}(s)=(n-2 \sigma)^{-1} a_{i}(s) / a_{0}(s)$, and $y(s)=\left(y_{1}(s), \cdots, y_{n}(s)\right)$. Then

$$
u(x+y, s)=\frac{2^{\frac{n-2 \sigma}{2}}}{|x|^{n-2 \sigma}}\left(a_{0}+\frac{\tilde{a}_{i j} x_{i} x_{j}}{|x|^{4}}+o\left(|x|^{-2}\right)\right)
$$

and

$$
\frac{\partial u}{\partial x_{i}}(x+y, s)=-\frac{(n-2 \sigma) a_{0} x_{i}}{|x|^{n-2 \sigma+2}}+O\left(|x|^{-(n-2 \sigma+3)}\right),
$$

where $\tilde{a}_{i j}=a_{i j}-\frac{n-2 \sigma}{2} \delta_{i j}-\frac{a_{i} a_{i}}{a_{0}}$. We only need to show that there exists a positive constant $C$ depending only on $n, \sigma, \inf _{\mathbb{S}^{n}} v(\cdot, 0)$ and $\|v(\cdot, 0)\|_{C^{3}\left(\mathbb{S}^{n}\right)}$ such that

$$
|y(s)| \leq C \quad \text { for all } 0 \leq s<T^{*} .
$$

Fix $T \in\left(0, T^{*}\right)$. After a rotation and a reflection, we may assume that $y_{n}(T)=\max _{i}\left|y_{i}(T)\right|$. From the Taylor expansions of $u$ and $\nabla u$ for $s=0$, we see that (e.g., Lemma 4.2 in [38]) there exists a $\lambda_{0}>0$, which depends only on $n, \sigma, \inf _{\mathbb{S}^{n}} v(\cdot, 0)$ and $\|v(\cdot, 0)\|_{C^{3}\left(\mathbb{S}^{n}\right)}$, such that for any $\lambda>\lambda_{0}$,

$$
u(x, 0)>u\left(x^{\lambda}, 0\right) \text { for } x_{n}<\lambda,
$$

where $x^{\lambda}=\left(x_{1}, \cdots, x_{n-1}, 2 \lambda-x_{n}\right)$. Denote $u^{\lambda}(x, s)=u\left(x^{\lambda}, s\right)$. By Proposition 2.3, we have

$$
u(x, s)>u^{\lambda}(x, s) \text { for all } s \in[0, T], x_{n}<\lambda, \lambda \geq \lambda_{0} .
$$

We claim that

$$
\max _{0 \leq s \leq T} y_{n}(s)<\lambda_{0} .
$$

If not, there exists $\bar{s} \in(0, T]$ such that $y_{n}(\bar{s})=\max _{0 \leq s \leq T} y_{n}(s) \geq \lambda_{0}$. Thus, we can set $\lambda=y_{n}(\bar{s})$ in (33), namely,

$$
u(x, s)>u^{\lambda}(x, s) \text { for all } s \in[0, T], x_{n}<\lambda=y_{n}(\bar{s}) .
$$

Let $\tilde{u}(x, s)=u\left(x+y_{n}(\bar{s}), s\right)$, then

$$
\tilde{u}\left(x^{\prime}, x_{n}, s\right)>\tilde{u}\left(x^{\prime},-x_{n}, s\right) \text { for all } s \in[0, T], x_{n}<0 .
$$

Let $\tilde{u}_{1}(x, s)=\frac{1}{|x|^{n-2 \sigma}} \tilde{u}\left(\frac{x}{|x|^{2}}, s\right)$. Then $\tilde{u}_{1}\left(x^{\prime}, x_{n}, s\right)$ and $\tilde{u}_{1}\left(x^{\prime},-x_{n}, s\right)$ satisfy (30) and

$$
\tilde{u}_{1}\left(x^{\prime}, x_{n}, s\right)>\tilde{u}_{1}\left(x^{\prime},-x_{n}, s\right) \text { for all } s \in[0, T], x_{n}<0 .
$$

By Proposition 2.3 .

$$
\left.\frac{\partial\left(\tilde{u}_{1}\left(x^{\prime}, x_{n}, s\right)-\tilde{u}_{1}\left(x^{\prime},-x_{n}, s\right)\right)}{\partial x_{n}}\right|_{(x, s)=(0, \bar{s})}<0
$$


i.e., $\left(\partial \tilde{u}_{1} / \partial x_{n}\right)(0, \bar{s})<0$. This contradicts (31). Hence, $\max _{0 \leq s \leq T} y_{n}(s)<\lambda_{0}$, which implies $y_{n}(T)<\lambda_{0}$. Since $\lambda_{0}$ is independent of $s$, we have $|y(s)| \leq \lambda_{0}$ for all $0 \leq s<T^{*}$. Moreover, $\lambda_{0}$ is independent of the choice of $q_{0}$, and we conclude that

$$
\sup _{\mathbb{S}^{n}} \frac{\left|\nabla_{\mathbb{S}^{n}} v\right|}{|v|} \leq C \quad \text { for all } s \in\left(0, T^{*}\right) .
$$

For each $t$, integrating the above inequality along a shortest geodesic between a maximum point and a minimum point of $v(\cdot, t)$ yields

$$
\max _{\mathbb{S}^{n}} v(\cdot, t) \leq C \min _{\mathbb{S}^{n}} v(\cdot, t),
$$

where $C$ depends only on $n, \sigma, \inf _{\mathbb{S}^{n}} v(\cdot, 0)$ and $\|v(\cdot, 0)\|_{C^{3}\left(\mathbb{S}^{n}\right)}$.

\section{Existence and convergence of a fractional Yamabe flow}

\subsection{Schauder estimates}

For an open set $\Omega \subset \mathbb{R}^{n}$ and $\gamma \in(0,1), C^{\gamma}(\Omega)$ denotes the standard Hölder space over $\Omega$, with the norm

$$
|v|_{\gamma ; \Omega}:=|v|_{0 ; \Omega}+[v]_{\gamma ; \Omega}:=\sup _{\Omega}|v(\cdot)|+\sup _{x_{1} \neq x_{2}, x_{1}, x_{2} \in \Omega} \frac{\left|u\left(x_{1}\right)-u\left(x_{2}\right)\right|}{\left|x_{1}-x_{2}\right|^{\gamma}} .
$$

For simplicity, we use $C^{\gamma}(\Omega)$ to denote $C^{[\gamma], \gamma-[\gamma]}(\Omega)$ when $1<\gamma \notin \mathbb{N}$ (the set of positive integers), where $[\gamma]$ is the integer part of $\gamma$. Since the operator $\partial_{t}+(-\Delta)^{\sigma}$ is invariant under the scaling $(x, t) \rightarrow\left(c x, c^{2 \sigma} t\right)$ with $c>0$, we introduce the fractional parabolic distance as

$$
\rho\left(X_{1}, X_{2}\right)=\left(\left|x_{1}-x_{2}\right|^{2}+\left|t_{1}-t_{2}\right|^{1 / \sigma}\right)^{1 / 2},
$$

where $X_{1}=\left(x_{1}, t_{2}\right), X_{2}=\left(x_{2}, t_{2}\right) \in \mathbb{R}^{n+1}$. For a measurable function $u$ defined in a Borel set $Q \subset \mathbb{R}^{n+1}$ and $0<\alpha<\min (1,2 \sigma)$, we define

$$
[u]_{\alpha, \frac{\alpha}{2 \sigma} ; Q}=\sup _{X_{1} \neq X_{2}, X_{1}, X_{2} \in Q} \frac{\left|u\left(X_{1}\right)-u\left(X_{2}\right)\right|}{\rho\left(X_{1}, X_{2}\right)^{\alpha}}
$$

and

$$
|u|_{\alpha, \frac{\alpha}{2 \sigma} ; Q}=|u|_{0 ; Q}+[u]_{\alpha, \frac{\alpha}{2 \sigma} ; Q},
$$

where $|u|_{0 ; Q}=\sup _{X \in Q}|u(X)|$. We denote $C^{\alpha, \frac{\alpha}{2 \sigma}}(Q)$ as the space of all measurable functions $u$ for which $|u|_{\alpha, \frac{\alpha}{2 \sigma} ; Q}<\infty$. Let $Q_{T}=\mathbb{R}^{n} \times(0, T], T \in(0, \infty)$. For $2 \sigma+\alpha \notin \mathbb{N}$ and $0<\alpha<\min (1,2 \sigma)$, we say $u \in \mathcal{C}^{2 \sigma+\alpha, 1+\frac{\alpha}{2 \sigma}}\left(Q_{T}\right)$ if

$$
[u]_{2 \sigma+\alpha, 1+\frac{\alpha}{2 \sigma} ; Q_{T}}:=\left[u_{t}\right]_{\alpha, \frac{\alpha}{2 \sigma} ; Q_{T}}+\left[(-\Delta)^{\sigma} u\right]_{\alpha, \frac{\alpha}{2 \sigma} ; Q_{T}}<\infty
$$


and

$$
|u|_{2 \sigma+\alpha, 1+\frac{\alpha}{2 \sigma} ; Q_{T}}:=|u|_{0 ; Q_{T}}+\left|u_{t}\right|_{0, Q_{T}}+\left|(-\Delta)^{\sigma} u\right|_{0 ; Q_{T}}+[u]_{2 \sigma+\alpha, 1+\frac{\alpha}{2 \sigma} ; Q_{T}}<\infty .
$$

Then $\mathcal{C}^{2 \sigma+\alpha, 1+\frac{\alpha}{2 \sigma}}\left(Q_{T}\right)$ is a Banach space equipped with the norm $|\cdot|_{2 \sigma+\alpha, 1+\frac{\alpha}{2 \sigma} ; Q_{T}}$.

Consider the following Cauchy problem

$$
\begin{cases}a(x, t) u_{t}+(-\Delta)^{\sigma} u+b(x, t) u=f(x, t), & \text { in } Q_{T}, \\ u(x, 0)=u_{0}(x), & \text { in } \mathbb{R}^{n},\end{cases}
$$

where $\lambda^{-1} \leq a(x, t) \leq \lambda$ for some constant $\lambda \geq 1$.

Lemma 4.1. Suppose $b(x, t)$ is bounded in $Q_{1}$. Let $u \in \mathcal{C}^{2 \sigma+\alpha, 1+\frac{\alpha}{2 \sigma}}\left(Q_{1}\right)$ satisfy

$$
\begin{cases}a(x, t) u_{t}+(-\Delta)^{\sigma} u+b(x, t) u \leq 0, & \text { in } Q_{1}, \\ u(x, 0) \leq 0, & \text { in } \mathbb{R}^{n},\end{cases}
$$

then $u \leq 0$ in $Q_{1}$.

Proof. Without loss of generality we may assume that $b(x, t) \geq 1$ as before. Let $\eta(x)$ be a smooth cut-off function supported in $B_{2} \subset \mathbb{R}^{n}$ and equal to 1 in $B_{1}$. Let $\eta_{R}(\cdot)=\eta(\cdot / R)$ and $v=\eta_{R} u$. Then

$$
a v_{t}+(-\Delta)^{\sigma} v+b(x, t) v \leq\left\langle u, \eta_{R}\right\rangle+u(-\Delta)^{\sigma} \eta_{R},
$$

where

$$
\langle u, \eta\rangle=c(n, \sigma) \int_{\mathbb{R}^{n}} \frac{(u(x, t)-u(y, t))(\eta(x, t)-\eta(y, t))}{|x-y|^{n+2 \sigma}} \mathrm{d} y .
$$

If $u$ is positive somewhere in $Q_{1}$, then we can choose $R$ as large as we want such that $v$ attains its positive maximum value in $Q_{1}$ at $\left(x_{0}, t_{0}\right) \in B_{R} \times(0,1]$. It is clear that $a\left(x_{0}, t_{0}\right) v_{t}\left(x_{0}, t_{0}\right)+$ $(-\Delta)^{\sigma} v\left(x_{0}, t_{0}\right) \geq 0$. Since $b \geq 1$, we have

$$
\sup _{B_{R} \times(0,1]} u \leq v\left(x_{0}, t_{0}\right) \leq \sup _{Q_{1}}\left|\left\langle u, \eta_{R}\right\rangle+u(-\Delta)^{\sigma} \eta_{R}\right| \rightarrow 0 \quad \text { as } R \rightarrow \infty .
$$

This finishes the proof of this Lemma.

Proposition 4.1. Let $0<\alpha<\min (1,2 \sigma)$ such that $2 \sigma+\alpha$ is not an integer. Suppose that $a(x, t), b(x, t), f(x, t) \in C^{\alpha, \frac{\alpha}{2 \sigma}}\left(Q_{1}\right)$ and $u_{0}(x) \in C^{2 \sigma+\alpha}\left(\mathbb{R}^{n}\right)$. Then there exists a unique solution $u \in \mathcal{C}^{2 \sigma+\alpha, 1+\frac{\alpha}{2 \sigma}}\left(Q_{1}\right)$ of (34). Moreover, there exists a constant $C>0$ depending only on $n, \sigma, \lambda, \alpha,|a|_{\alpha, \frac{\alpha}{2 \sigma} ; Q_{1}}$ and $|b|_{\alpha, \frac{\alpha}{2 \sigma} ; Q_{1}}$ such that

$$
|u|_{2 \sigma+\alpha, 1+\frac{\alpha}{2 \sigma} ; Q_{1}} \leq C\left(\left|u_{0}\right|_{2 \sigma+\alpha ; \mathbb{R}^{n}}+|f|_{\alpha, \frac{\alpha}{2 \sigma} ; Q_{1}}\right) .
$$


Proof. By Lemma 4.1 there exists $C>0$ depending only on $\lambda,|b|_{L^{\infty}\left(Q_{1}\right)}$ such that

$$
|u|_{0 ; Q_{1}} \leq C\left(\left|u_{0}\right|_{0 ; \mathbb{R}^{n}}+|f|_{0 ; Q_{1}}\right)
$$

Then the uniqueness of solutions of (34) follows immediately. In the following, we will show the a priori estimate (36). By (37) and some interpolation inequalities in Lemma B.1, we only need to show, instead of (36),

$$
[u]_{2 \sigma+\alpha, 1+\frac{\alpha}{2 \sigma} ; Q_{1}} \leq C\left(\left|u_{0}\right|_{2 \sigma+\alpha ; \mathbb{R}^{n}}+|f|_{\alpha, \frac{\alpha}{2 \sigma} ; Q_{1}}\right) .
$$

First of all, (38) holds provided $a=1, b=0$ (see, e.g., [59]), and it can be easily extended to the case that $a$ is a positive constant. For the general case, we use the "freezing coefficients" method (see, e.g., [56]).

Fix a small $\delta>0$, which will be specified later. We can find two points $X_{1}, X_{2} \in Q_{1}$ such that

$$
\frac{\left|u_{t}\left(X_{1}\right)-u_{t}\left(X_{2}\right)\right|}{\rho\left(X_{1}, X_{2}\right)^{\alpha}} \geq \frac{1}{2}\left[u_{t}\right]_{\alpha, \frac{\alpha}{2 \sigma} ; Q_{1}} .
$$

If $\rho\left(X_{1}, X_{2}\right)>\delta$, then

$$
\left[u_{t}\right]_{\alpha, \frac{\alpha}{2 \sigma} ; Q_{1}} \leq 4 \delta^{-\alpha}\left|u_{t}\right|_{0 ; Q_{1}} .
$$

It follows from Lemma B.1 that, for any small $\varepsilon_{0}>0$,

$$
\left[u_{t}\right]_{\alpha, \frac{\alpha}{2 \sigma} ; Q_{1}} \leq \varepsilon_{0}[u]_{2 \sigma+\alpha, 1+\frac{\alpha}{2 \sigma} ; Q_{1}}+C_{0}|u|_{0 ; Q_{1}},
$$

where $C_{0}>0$ depends on $n, \sigma, \alpha, \varepsilon_{0}, \delta$.

If $\rho\left(X_{1}, X_{2}\right) \leq \delta$, take a cut-off function $\eta(X) \in C^{\infty}\left(\mathbb{R}^{n+1}\right)$ such that $\eta(X)=1$ for $\rho\left(X, X_{1}\right) \leq \delta, \eta(X)=0$ for $\rho\left(X, X_{1}\right) \geq 2 \delta$. By the estimates of solutions of (34) with $a$ being a positive constant and $b \equiv 0$, we have

$$
\begin{aligned}
{\left[u_{t}\right]_{\alpha, \frac{\alpha}{2 \sigma} ; Q_{1}} } & \leq 2 \frac{\left|u_{t}\left(X_{1}\right)-u_{t}\left(X_{2}\right)\right|}{\rho\left(X_{1}, X_{2}\right)^{\alpha}} \leq 2[u \eta]_{2 \sigma+\alpha, 1+\frac{\alpha}{2 \sigma} ; Q_{1}} \\
& \leq C_{1}\left(\left|a\left(X_{1}\right)(u \eta)_{t}+(-\Delta)^{\sigma}(u \eta)\right|_{\alpha, \frac{\alpha}{2 \sigma} ; Q_{1}}+\left|u_{0} \eta\right|_{2 \sigma+\alpha ; \mathbb{R}^{n}}+|u \eta|_{0 ; Q_{1}}\right),
\end{aligned}
$$

where $C_{1}>0$ is independent of $\delta$. Note that

$$
\begin{aligned}
& a\left(X_{1}\right)(u \eta)_{t}+(-\Delta)^{\sigma}(u \eta) \\
& =\eta\left(a(X) u_{t}+(-\Delta)^{\sigma} u\right)+\eta\left(a\left(X_{1}\right)-a(X)\right) u_{t}+a\left(X_{1}\right) u \eta_{t}-\langle u, \eta\rangle+u(-\Delta)^{\sigma} \eta \\
& =\eta(f-b u)+\eta\left(a\left(X_{1}\right)-a(X)\right) u_{t}+a\left(X_{1}\right) u \eta_{t}-\langle u, \eta\rangle+u(-\Delta)^{\sigma} \eta,
\end{aligned}
$$

where $\langle u, \eta\rangle$ is defined in (35). Since $\left|\eta(X)\left(a\left(X_{1}\right)-a(X)\right)\right| \leq[a]_{\alpha, \frac{\alpha}{2 \sigma} ; Q_{1}} \delta^{\alpha}$, making use of Lemma B.1 again, we have

$$
\begin{aligned}
{\left[u_{t}\right]_{\alpha, \frac{\alpha}{2 \sigma} ; Q_{1}} \leq } & C_{1} \delta^{\alpha}[u]_{2 \sigma+\alpha, 1+\frac{\alpha}{2 \sigma} ; Q_{1}}+C(\delta)\left(|u|_{0 ; Q_{1}}+|f|_{\alpha, \frac{\alpha}{2 \sigma} ; Q_{1}}\right) \\
& +C_{1}|\langle u, \eta\rangle|_{\alpha, \frac{\alpha}{2 \sigma} ; Q_{1}}+C_{1}\left|u_{0} \eta\right|_{2 \sigma+\alpha ; \mathbb{R}^{n}} .
\end{aligned}
$$


Hence, from (62) in Lemma B.3, (40) and (39), we can conclude that

$$
\left[u_{t}\right]_{\alpha, \frac{\alpha}{2 \sigma} ; Q_{1}} \leq\left(C_{1} \delta^{\alpha}+\varepsilon_{0}\right)[u]_{2 \sigma+\alpha, 1+\frac{\alpha}{2 \sigma} ; Q_{1}}+C(\delta)\left(|u|_{0, Q_{1}}+|f|_{\alpha, \frac{\alpha}{2 \sigma} ; Q_{1}}+\left|u_{0}\right|_{2 \sigma+\alpha ; \mathbb{R}^{n}}\right) .
$$

Since

$$
u_{t}+(-\Delta)^{\sigma} u=(1-a) u_{t}-b u+f
$$

we see that

$$
[u]_{2 \sigma+\alpha, 1+\frac{\alpha}{2 \sigma} ; Q_{1}} \leq C\left(\left[u_{t}\right]_{\alpha, \frac{\alpha}{2 \sigma} ; Q_{1}}+|u|_{0 ; Q_{1}}+|f|_{\alpha, \frac{\alpha}{2 \sigma} ; Q_{1}}+\left|u_{0}\right|_{2 \sigma+\alpha ; \mathbb{R}^{n}},\right),
$$

where $C>0$ depending only on $n, \sigma, \lambda, \alpha,\|a\|_{\alpha, \frac{\alpha}{2 \sigma} ; Q_{1}}$ and $\|a, b\|_{\alpha, \frac{\alpha}{2 \sigma} ; Q_{1}}$. Then (36) follows from (37), (42) and (41) by choosing sufficiently small $\delta$ and $\varepsilon_{0}$.

Finally, the existence of solutions of (34) follows from standard continuity method.

Remark 4.1. Cauchy problems for non-local operators and pseudo-differential operators in different spaces have been studied, e.g., in [55], [62], [63], [64] and references therein.

Remark 4.2. Observe that in the proof of the above proposition the only place we use the uniform lower and upper bounds of $a(x)$ is that at $X_{1}$, that is $\frac{1}{\lambda} \leq a\left(X_{1}\right) \leq \lambda$. This observation will be used in the proof of Proposition 4.2

Remark 4.3. One can also obtain the estimates in $Q_{T}$ by considering the scaled function $\tilde{u}(x, t):=u\left(T^{1 / 2 \sigma} x, T t\right)$.

For $\gamma \in(0,1), C^{\gamma}\left(\mathbb{S}^{n}\right)$ denotes the standard Hölder space over $\mathbb{S}^{n}$, with norm

$$
|v|_{\gamma ; \mathbb{S}^{n}}:=|v|_{0 ; \mathbb{S}^{n}}+[v]_{\gamma ; \mathbb{S}^{n}}:=\sup _{\mathbb{S}^{n}}|v(\cdot)|+\sup _{\xi_{1} \neq \xi_{2}, \xi_{1}, \xi_{2} \in \mathbb{S}^{n}} \frac{\left|v\left(\xi_{1}\right)-u\left(\xi_{2}\right)\right|}{\left|\xi_{1}-\xi_{2}\right|^{\gamma}},
$$

where $\left|\xi_{1}-\xi_{2}\right|$ is understood as the Euclidean distance from $\xi_{1}$ to $\xi_{2}$ in $\mathbb{R}^{n+1}$. For simplicity, we use $C^{\gamma}\left(\mathbb{S}^{n}\right)$ to denote $C^{[\gamma], \gamma-[\gamma]}\left(\mathbb{S}^{n}\right)$ when $1<\gamma \notin \mathbb{N}$, where $[\gamma]$ is the integer part of $\gamma$. For $Y_{1}=\left(\xi_{1}, t_{1}\right), Y_{2}=\left(\xi_{2}, t_{2}\right) \in \mathbb{S}^{n} \times(0, \infty)$ we denote

$$
\rho\left(Y_{1}, Y_{2}\right)=\left(\left|\xi_{1}-\xi_{2}\right|^{2}+\left|t_{1}-t_{2}\right|^{1 / \sigma}\right)^{1 / 2} .
$$

We still assume that $0<\alpha<\min (1,2 \sigma)$. Let $\mathcal{Q}_{T}=\mathbb{S}^{n} \times(0, T]$ for $T>0$. We say $v \in C^{\alpha, \frac{\alpha}{2 \sigma}}\left(\mathcal{Q}_{T}\right)$ if

$$
|v|_{\alpha, \frac{\alpha}{2 \sigma} ; \mathcal{Q}_{T}}=|v|_{0 ; \mathcal{Q}_{T}}+[v]_{\alpha, \frac{\alpha}{2 \sigma} ; \mathcal{Q}_{T}}:=\sup _{Y \in \mathcal{Q}_{T}} v(Y)+\sup _{Y_{1} \neq Y_{2}, Y_{1}, Y_{2} \in \mathcal{Q}_{T}} \frac{\left|v\left(Y_{1}\right)-u\left(Y_{2}\right)\right|}{\rho\left(Y_{1}, Y_{2}\right)^{\alpha}}<\infty,
$$

and $v \in \mathcal{C}^{2 \sigma+\alpha, 1+\frac{\alpha}{2 \sigma}}\left(\mathcal{Q}_{T}\right)$ if

$$
[v]_{2 \sigma+\alpha, 1+\frac{\alpha}{2 \sigma} ; \mathcal{Q}_{T}}:=\left[v_{t}\right]_{\alpha, \frac{\alpha}{2 \sigma} ; \mathcal{Q}_{T}}+\left[P_{\sigma}(v)\right]_{\alpha, \frac{\alpha}{2 \sigma} ; \mathcal{Q}_{T}}<\infty
$$


and

$$
|v|_{2 \sigma+\alpha, 1+\frac{\alpha}{2 \sigma} ; \mathcal{Q}_{T}}:=|v|_{0 ; \mathcal{Q}_{T}}+\left|v_{t}\right|_{0, \mathcal{Q}_{T}}+\left|P_{\sigma}(v)\right|_{0 ; \mathcal{Q}_{T}}+[v]_{2 \sigma+\alpha, 1+\frac{\alpha}{2 \sigma} ; \mathcal{Q}_{T}}<\infty .
$$

Then $\mathcal{C}^{2 \sigma+\alpha, 1+\frac{\alpha}{2 \sigma}}\left(\mathcal{Q}_{T}\right)$ is a Banach space equipped with the norm $|\cdot|_{2 \sigma+\alpha, 1+\frac{\alpha}{2 \sigma} ; \mathcal{Q}_{T}}$.

Proposition 4.2. Let $0<\alpha<\min (1,2 \sigma)$ such that $2 \sigma+\alpha$ is not an integer. Let $a(\xi, t)$, $b(\xi, t), f(\xi, t) \in C^{\alpha, \frac{\alpha}{2 \sigma}}\left(\mathcal{Q}_{1}\right), v_{0} \in C^{2 \sigma+\alpha}\left(\mathbb{S}^{n}\right)$ and $\lambda^{-1} \leq a(\xi, t) \leq \lambda$ for some $\lambda \geq 1$. Then there exists a unique function $v \in \mathcal{C}^{2 \sigma+\alpha, 1+\frac{\alpha}{2 \sigma}}\left(\mathcal{Q}_{1}\right)$ such that

$$
\begin{cases}a v_{t}+P_{\sigma}(v)+b v=f, & \text { in } \mathcal{Q}_{1}, \\ v(y, 0)=v_{0}(y), & \text { in } \mathbb{S}^{n} .\end{cases}
$$

Moreover, there exists a constant $C$ depending only on $n, \sigma, \lambda, \alpha,|a|_{\alpha, \frac{\alpha}{2 \sigma} ; \mathcal{Q}_{1}}$ and $|b|_{\alpha, \frac{\alpha}{2 \sigma} ; \mathcal{Q}_{1}}$ such that

$$
|v|_{2 \sigma+\alpha, 1+\frac{\alpha}{2 \sigma} ; \mathcal{Q}_{1}} \leq C\left(\left|v_{0}\right|_{2 \sigma+\alpha ; \mathbb{S}^{n}}+|f|_{\alpha, \frac{\alpha}{2 \sigma} ; \mathcal{Q}_{1}}\right) .
$$

Proof. Uniqueness of solutions of (43) follows from maximum principles. We only need to show a priori estimate (44), from which the existence of solution of (43) follows by the standard continuity method.

Choose $Y_{1}=\left(\xi_{1}, t_{1}\right), Y_{2}=\left(\xi_{2}, t_{2}\right) \in \mathbb{S}^{n} \times(0, T)$ such that

$$
\frac{\left|v_{t}\left(Y_{1}\right)-v_{t}\left(Y_{2}\right)\right|}{\rho\left(Y_{1}, Y_{2}\right)^{\alpha}} \geq \frac{1}{2}\left[v_{t}\right]_{\alpha, \frac{\alpha}{2 \sigma} ; \mathcal{Q}_{1}} .
$$

Without loss of generality we may assume that $\xi_{1}, \xi_{2}$ are on the south hemisphere. Let $F(x)$ be the inverse of stereographic projection from the north pole and

$$
u(x, t)=\left(\frac{2}{1+|x|^{2}}\right)^{\frac{n-2 \sigma}{2}} v(F(x), t) .
$$

There exist $x_{1}, x_{2} \in B(0,1)$ such that $Y_{1}=\left(F\left(x_{1}\right), t_{1}\right), Y_{2}=\left(F\left(x_{2}\right), t_{2}\right)$. We denote $X_{1}=$ $\left(x_{1}, t_{1}\right), X_{2}=\left(x_{2}, t_{2}\right)$. By (45) there exists a constant $C$ depending only $n, \sigma, \alpha$ such that

$$
\left[u_{t}\right]_{\alpha, \frac{\alpha}{2 \sigma} ; Q_{1}} \leq C\left|v_{t}\right|_{0, \mathcal{Q}_{1}}+C\left|u_{t}\right|_{0, Q_{1}}+C \frac{\left|u_{t}\left(X_{1}\right)-u_{t}\left(X_{2}\right)\right|}{\rho\left(X_{1}, X_{2}\right)^{\alpha}} .
$$

Note that $u$ satisfies (34) with $a, b, f$ replaced by

$$
\left(\frac{2}{1+|x|^{2}}\right)^{2 \sigma} a(F(x), t),\left(\frac{2}{1+|x|^{2}}\right)^{2 \sigma} b(F(x), t),\left(\frac{2}{1+|x|^{2}}\right)^{\frac{n+2 \sigma}{2}} f(F(x), t) .
$$

In view of Remark 4.2 and the arguments in the proof of Proposition 4.1, we conclude that

$$
[u]_{2 \sigma+\alpha, 1+\frac{\alpha}{2 \sigma} ; Q_{1}} \leq C\left(\left|v_{0}\right|_{2 \sigma+\alpha ; \mathbb{S}^{n}}+|v|_{0 ; \mathcal{Q}_{1}}+\left|v_{t}\right|_{0 ; \mathcal{Q}_{1}}+|f|_{\alpha, \frac{\alpha}{2 \sigma} ; \mathcal{Q}_{1}}\right) .
$$


Hence, together with (45) and interpolation inequalities in Lemma B.2, we have

$$
\left[v_{t}\right]_{\alpha, \frac{\alpha}{2 \sigma} ; \mathcal{Q}_{1}} \leq C\left(\left|v_{0}\right|_{2 \sigma+\alpha ; \mathbb{S}^{n}}+|v|_{0 ; \mathcal{Q}_{1}}+|f|_{\alpha, \frac{\alpha}{2 \sigma} ; \mathcal{Q}_{1}}\right) .
$$

It follows from the maximum principle that $|v|_{0 ; \mathcal{Q}_{1}} \leq C\left(\left|v_{0}\right|_{2 \sigma+\alpha ; \mathbb{S}^{n}}+|f|_{\alpha, \frac{\alpha}{2 \sigma} ; \mathcal{Q}_{1}}\right)$. Hence (44) follows from (46), (43) and some inequalities in Lemma B.2

Corollary 4.1. Let $0<\alpha<\min (1,2 \sigma)$ such that $2 \sigma+\alpha$ is not an integer. Let $a(\xi, t), b(\xi, t)$, $f(\xi, t) \in C^{\alpha, \frac{\alpha}{2 \sigma}}\left(\mathcal{Q}_{3}\right), \lambda^{-1} \leq a(\xi, t) \leq \lambda$ for some $\lambda \geq 1$. Suppose that $v \in \mathcal{C}^{2 \sigma+\alpha, 1+\frac{\alpha}{2 \sigma}}\left(\mathcal{Q}_{3}\right)$ satisfies

$$
a v_{t}+P_{\sigma}(v)+b v=f, \quad \text { in } \mathcal{Q}_{3} .
$$

Then there exists a constant $C$ depending only on $n, \sigma, \lambda, \alpha,|a|_{\alpha, \frac{\alpha}{2 \sigma} ; \mathbb{S}^{n} \times[1,3]}$ and $|b|_{\alpha, \frac{\alpha}{2 \sigma} ; \mathbb{S}^{n} \times[1,3]}$ such that

$$
|v|_{2 \sigma+\alpha, 1+\frac{\alpha}{2 \sigma} ; \mathbb{S}^{n} \times[2,3]} \leq C\left(|v|_{\alpha, \frac{\alpha}{2 \sigma} ; \mathbb{S}^{n} \times[1,3]}+|f|_{\alpha, \frac{\alpha}{2 \sigma} ; \mathbb{S}^{n} \times[1,3]}\right) .
$$

Proof. Let $\eta(t)$ be a smooth cut-off function defined on $\mathbb{R}$ such that $\eta(t)=0$ when $t \leq 4 / 3$ and $\eta(t)=1$ when $t \geq 5 / 3$. Then $\tilde{v}:=\eta v$ satisfies

$$
\left\{\begin{array}{l}
a \tilde{v}_{t}+P_{\sigma}(\tilde{v})+b \tilde{v}=f \eta+a v \eta_{t}, \quad \text { in } \mathbb{S}^{n} \times[1,3], \\
\tilde{v}(\cdot, 1)=0
\end{array}\right.
$$

The Corollary follows immediately from Proposition 4.2 ,

\subsection{Short time existence}

Proposition 4.3. Let $0<\alpha<\min (1,2 \sigma)$ such that $2 \sigma+\alpha$ is not an integer. Let $v_{0} \in$ $C^{2 \sigma+\alpha}\left(\mathbb{S}^{n}\right)$ and $v_{0}>0 \mathrm{in} \mathbb{S}^{n}$. Then there exists a small positive constant $T_{*}$ depending only on $n, \sigma, \alpha, \inf _{\mathbb{S}^{n}} v_{0},\left|v_{0}\right|_{2 \sigma+\alpha ; \mathbb{S}^{n}}$ and a unique positive solution $v \in \mathcal{C}^{2 \sigma+\alpha, 1+\frac{\alpha}{2 \sigma}}\left(\mathbb{S}^{n} \times\left[0, T_{*}\right]\right)$ of (8) in $\mathbb{S}^{n} \times\left(0, T_{*}\right]$ with $v(\cdot, 0)=v_{0}$. Furthermore, $v$ is smooth in $\mathbb{S}^{n} \times\left(0, T_{*}\right)$.

Proof. By a scaling argument in the time variable, we only need to show the short time existence of

$$
\left\{\begin{array}{l}
\frac{\partial v^{N}}{\partial t}=-P_{\sigma}(v) \\
v(\cdot, 0)=v_{0}
\end{array}\right.
$$

We shall use the Implicit Function Theorem. By Proposition 4.2, there exists a function $w \in \mathcal{C}^{2 \sigma+\alpha, 1+\frac{\alpha}{2 \sigma}\left(\mathbb{S}^{n} \times(0,1]\right) \text { such that }}$

$$
\left\{\begin{array}{l}
N v_{0}^{N-1} w_{t}=-P_{\sigma}(w), \quad \text { in } \mathbb{S}^{n} \times(0,1], \\
w(\cdot, 0)=v_{0}
\end{array}\right.
$$

and for any small positive constant $\varepsilon_{0}$, we have $\left\|w(\cdot, t)-v_{0}\right\|_{C^{2 \sigma+\alpha}\left(\mathbb{S}^{n}\right)} \leq \varepsilon_{0}$ provided $t \leq T_{\varepsilon_{0}}$. Here $T_{\varepsilon_{0}}$ is a positive constant depending on $\varepsilon_{0}$. Hence, we may assume that $w>0$ in $\mathbb{S}^{n}$. 
Denote

$$
\mathscr{X}=\left\{\varphi \in \mathcal{C}^{2 \sigma+\alpha, 1+\frac{\alpha}{2 \sigma}}\left(\mathbb{S}^{n} \times\left(0, T_{\varepsilon_{0}}\right]\right): \varphi(\cdot, 0)=0\right\},
$$

and

$$
\mathscr{Y}=C^{\alpha, \frac{\alpha}{2 \sigma}}\left(\mathbb{S}^{n} \times\left(0, T_{\varepsilon_{0}}\right]\right) .
$$

Define $\mathcal{F}(v):=N|v|^{N-1} \frac{\partial v}{\partial t}+P_{\sigma}(v)$ for $v \in \mathcal{C}^{2 \sigma+\alpha, 1+\frac{\alpha}{2 \sigma}}\left(\mathbb{S}^{n} \times\left(0, T_{\varepsilon_{0}}\right]\right)$, and

$$
L: \mathscr{X} \rightarrow \mathscr{Y}, \quad \varphi \mapsto \mathcal{F}(w+\varphi)-\mathcal{F}(w) .
$$

Note that $L(0)=0$,

$$
L^{\prime}(0) \varphi=N w^{N-1} \varphi_{t}+P_{\sigma}(\varphi)+N(N-1) w^{N-2} w_{t} \varphi, \quad \forall \varphi \in \mathscr{X} .
$$

It follows from Proposition 4.2 that $L^{\prime}(0): \mathscr{X} \rightarrow \mathscr{Y}$ is invertible when $\varepsilon_{0}$ is chosen sufficiently small.

By the Implicit Function Theorem, there exists a positive constant $\delta>0$ such that for any $\phi \in \mathscr{Y}$ with $\|\phi\|_{\mathscr{Y}} \leq \delta$ there exists a unique solution $\varphi \in \mathscr{X}$ of the equation

$$
L(\varphi)=\phi .
$$

Let $T_{*}>0$ be small. Pick a cut off function $0 \leq \eta(t) \leq 1$ in $\mathbb{R}_{+}$satisfying $\eta(t)=1$ for $s \leq T_{*}$ and $\eta(t)=0$ if $s \geq 2 T_{*}$. It is easy to see that

$$
\|\eta(t) \mathcal{F}(w)\|_{\mathscr{Y}} \leq \delta
$$

provided $T_{*}$ is sufficiently small. Therefore, there exists a function $\varphi \in \mathscr{X}$ such that

$$
L(\varphi)=-\eta(t) \mathcal{F}(w) .
$$

Thus, $v:=w+\varphi$ satisfies $v(\cdot, 0)=v_{0}$ and

$$
\mathcal{F}(w+\varphi)=0, \quad \text { in } \mathbb{S}^{n} \times\left(0, T_{*}\right] .
$$

Moreover, $v$ is positive if $T_{*}$ is small enough. The smoothness of $v$ follows from Corollary 4.1 and bootstrap arguments.

\subsection{Long time existence and convergence}

Proposition 4.4. Let $v$ be a positive smooth solution of $\left[8\right.$ in $\mathbb{S}^{n} \times(0,3]$ and satisfy $\Lambda^{-1} \leq$ $v(y, t) \leq \Lambda$ for all $(y, t) \in \mathbb{S}^{n} \times(0,3]$ with some positive constant $\Lambda$. Then for any positive integer $k$,

$$
\|v\|_{C^{k}\left(\mathbb{S}^{n} \times[2,3]\right)} \leq C
$$

where $C>0$ depends only on $n, \sigma, k, \Lambda$, and $r_{\sigma}^{g(1)}$. 
Proof. We first observe that $r_{\sigma}^{g(t)}$ is decreasing in $t$, and is lower bounded away from 0 by Sobolev inequalities (see, e.g., [5]). Hence through a scaling argument in $t$, we may assume that $v$ satisfies the equation $\frac{\partial v^{N}}{\partial t}=-P_{\sigma}(v)$ instead of (8). By the Hölder estimates in [2] (see also Theorem 9.3 in [29]), there exists some $\beta \in(0, \min (1,2 \sigma))$ such that

$$
|v|_{\beta, \frac{\beta}{2 \sigma} ; \mathbb{S}^{n} \times[1,3]} \leq C(n, \sigma, \beta, \Lambda) .
$$

The Proposition follows from Corollary 4.1 and bootstrap arguments.

Proof of Theorem 1.1 By Proposition 4.3, we have a unique positive smooth solution of (7) on a maximum time interval $\left[0, T^{*}\right)$. Since the flow preserves the volume of the sphere, the Harnack inequality in Theorem 3.1 implies that $v(x, t)$ is uniformly bounded from above and away from zero. Proposition 4.4 yields smooth estimates for $v$ on $\mathbb{S}^{n} \times\left[\min \left(1, T^{*} / 2\right), T^{*}\right)$. It follows that $T^{*}=\infty$, since otherwise by Proposition 4.3 we can extend $v$ beyond $T^{*}$. Moreover, there exists $v_{\infty} \in C^{\infty}\left(\mathbb{S}^{n}\right)$ and a sequence $\left\{v\left(t_{j}\right)\right\}$ such that $v\left(t_{j}\right)$ converges smoothly to $v_{\infty}$. By Theorem A.4 in the Appendix, $v(t)$ converges smoothly to $v_{\infty}$, i.e. there exists a smooth metric $g_{\infty}$ on $\mathbb{S}^{n}$ such that $g(t)$ converges smoothly to $g_{\infty}$. The formula for the gradient of the total $\sigma$-curvature gives

$$
\frac{d S}{d t}=-\frac{n-2 \sigma}{2 n}\left(\operatorname{vol}_{g}\left(\mathbb{S}^{n}\right)\right)^{\frac{2 \sigma-n}{n}} \int_{\mathbb{S}^{n}}\left(R_{\sigma}^{g}-r_{\sigma}^{g}\right)^{2} \mathrm{~d} v o l_{g}
$$

Thus,

$$
\int_{0}^{\infty} \int_{\mathbb{S}^{n}}\left(R_{\sigma}^{g}-r_{\sigma}^{g}\right)^{2} \mathrm{~d} v o l_{g}<\infty
$$

which implies that $R_{\sigma}^{g_{\infty}}$ is a positive constant.

\section{Extinction profile of a fractional porous medium equation}

Let $u(x, t)$ be the solution of (11) and $T>0$ be its extinction time. Since $u_{0}$ is not identically zero, it is proved in [29] that $u(x, t)>0$ in $\mathbb{R}^{n} \times(0, T)$ and $u(x, t) \in C^{\alpha}\left(\mathbb{R}^{n} \times(0, T)\right)$ for some $\alpha \in(0,1)$. We define $v(F(x), s)$ for all $x \in \mathbb{R}^{n}$ and all $s \geq 0$ as

$$
v(F(x), s):=\left.\left(\frac{1+|x|^{2}}{2}\right)^{\frac{n-2 \sigma}{2}}(T-t)^{-m /(1-m)} u(x, t)^{m}\right|_{t=T\left(1-e^{-s}\right)},
$$

where $F: \mathbb{R}^{n} \rightarrow \mathbb{S}^{n}$ is the inverse of stereographic projection from the north pole and $m=$ $\frac{n-2 \sigma}{n+2 \sigma}$. By the assumption of $u_{0}$, we have $v(\cdot, 0) \in C^{2}\left(\mathbb{S}^{n}\right)$. It follows from Proposition 4.3 that, there exists an $s^{*}>0$ and a unique positive function $\tilde{v} \in C^{\infty}\left(\mathbb{S}^{n} \times\left(0, s^{*}\right)\right)$ satisfies

$$
\frac{\partial \tilde{v}^{N}}{\partial s}=-P_{\sigma}(\tilde{v})+\frac{1}{1-m} \tilde{v}^{N}
$$


and $\tilde{v}_{0}=v(\cdot, 0)$. On the other hand, $\tilde{u}(x, t)$, which is defined by $\tilde{v}$ through (48), satisfies (111). By the uniqueness theorem on the solution of (11) in [29], $v \equiv \tilde{v}$ in $\mathbb{S}^{n} \backslash\{\mathcal{N}\} \times\left(0, s^{*}\right)$, and hence $v$ can be extended to a positive and smooth function in $\mathbb{S}^{n} \times\left(0, s^{*}\right)$.

Our first goal is that $v$ defined by relation (48) is positive and smooth in $\mathbb{S}^{n} \times(0, \infty)$. Secondly, we will show that $v$ converges to a steady solution of 499. In summary, we will show the following theorem in terms of $v$.

Theorem 5.1. Let $v$ be defined by relation (48). Then $v$ is positive and smooth in $\mathbb{S}^{n} \times(0, \infty)$. Moreover, there is a unique positive solution $\bar{v}$ of

$$
-P_{\sigma}(\bar{v})+\frac{1}{1-m} \bar{v}^{N}=0
$$

such that

$$
\|v(y, s)-\bar{v}(y)\|_{C^{3}\left(\mathbb{S}^{n}\right)} \rightarrow 0 \quad \text { as } s \rightarrow \infty .
$$

Our proof of Theorem 5.1 is inspired by some arguments in [30]. To prove convergence of $v(\cdot, t)$, we first establish the following universal estimates.

Proposition 5.1. Let $v$ be defined by relation (48). There exist positive constants $\beta_{1}, \beta_{2}$ such that

$$
\beta_{1} \leq v(y, s) \leq \beta_{2}
$$

for all $y \in \mathbb{S}^{n}, s^{*} / 2 \leq s<+\infty$. Hence, $v \in C^{\infty}\left(\mathbb{S}^{n} \times\left(s^{*} / 2, \infty\right)\right)$.

Proof. Step1: We show that if $s_{0}$ is such that $v$ is positive and smooth in $\mathbb{S}^{n} \times\left(s^{*} / 2, s_{0}\right)$, then there is a positive constant $\kappa_{1}$, independent of $s_{0}$, such that for all $s \in\left(s^{*} / 2, s_{0}\right)$

$$
\max _{\mathbb{S}^{n}} v(\cdot, s)>\kappa_{1}
$$

Let us argue by contradiction. If this is not true, then for every small $\varepsilon>0$, there is an $s_{\varepsilon}$ such that $s_{0}>s_{\varepsilon}>s^{*} / 2$ and $v\left(y, s_{\varepsilon}\right)<\varepsilon$ for all $y \in \mathbb{S}^{n}$. Given $\varepsilon>0$, consider

$$
U(x, t)=K^{1 / m}\left[\left(1+s_{\varepsilon}-\log T+\log (T-t)\right)(T-t)\right]_{+}^{\frac{1}{1-m}}\left(\frac{2}{1+|x|^{2}}\right)^{\frac{n+2 \sigma}{2}},
$$

where $K$ will be chosen later. Direct computations yield that

$$
\begin{aligned}
U_{t}- & (\Delta)^{\sigma} U^{\frac{n-2 \sigma}{n+2 \sigma}} \\
= & K^{\frac{1}{m}}\left[\left(1+s_{\varepsilon}-\log T+\log (T-t)\right)(T-t)\right]_{+}^{\frac{m}{1-m}}\left(\frac{2}{1+|x|^{2}}\right)^{\frac{n+2 \sigma}{2}} \\
& \cdot\left(\log T-\log (T-t)-2-s_{\varepsilon}+P_{\sigma}(1) K^{1-1 / m}\right),
\end{aligned}
$$


where we used that $(-\Delta)^{\sigma}\left(\frac{2}{1+|x|^{2}}\right)^{\frac{n-2 \sigma}{2}}=P_{\sigma}(1)\left(\frac{2}{1+|x|^{2}}\right)^{\frac{n+2 \sigma}{2}}$ with $P_{\sigma}(1)$ given in (4).

Let $t_{\varepsilon}$ be that $s_{\varepsilon}-\log T+\log \left(T-t_{\varepsilon}\right)=0$. We choose $K$ small such that $P_{\sigma}(1) K^{\frac{m-1}{m}}>2$ and let $\varepsilon=K$. Since $v\left(y, s_{\varepsilon}\right)<\varepsilon$,

$$
u\left(x, t_{\varepsilon}\right)<\varepsilon^{1 / m}\left(T-t_{\varepsilon}\right)^{\frac{1}{1-m}}\left(\frac{2}{1+|x|^{2}}\right)^{\frac{n+2 \sigma}{2}}=U\left(x, t_{\varepsilon}\right) .
$$

For $t>t_{\varepsilon}, U(x, t)$ is a supersolution of (11). It follows from the comparison principle (see the proof of Theorem 6.2 in [29]) that $u(x, t) \leq U(x, t)$. But $U$ vanishes before $T$. Hence, $u$ vanishes before $T$, which contradicts the definition of the extinction time $T$.

Step 2: $v$ is strictly positive and smooth for $s^{*} / 2<s<\infty$.

To show this, we define

$$
s_{0}=\sup \left\{s>0: v \in C^{3,1}\left(\mathbb{S}^{n} \times\left(s^{*} / 2, s\right)\right)\right\} .
$$

Note that $s_{0} \geq s^{*}$. We assume that $s_{0}<\infty$. Since $v \in C^{3,1}\left(\mathbb{S}^{n} \times\left(s^{*} / 2, s_{0}\right)\right)$ and $v$ is positive, by Theorem 3.1 and step 1 we have that $v$ is uniformly lower bounded away from 0 . We define

$$
U(x, t)=(M-t)_{+}^{1 /(1-m)} k(n, \sigma)\left(\frac{1}{1+|x|^{2}}\right)^{\frac{n+2 \sigma}{2}},
$$

where $k(n, \sigma)$ is defined in Theorem 1.3. $U(x, t)$ satisfies (11) and will be used as a barrier function. By our assumptions on $u_{0}$, we choose sufficiently large $M>T$ such that

$$
u_{0}(x) \leq M^{1 /(1-m)} k(n, \sigma)\left(\frac{1}{1+|x|^{2}}\right)^{\frac{n+2 \sigma}{2}} .
$$

It follows from comparison principle (Theorem 6.2 in [29]) that for all $0<t<T$,

$$
u(x, t) \leq(M-t)^{1 /(1-m)} k(n, \sigma)\left(\frac{1}{1+|x|^{2}}\right)^{\frac{n+2 \sigma}{2}} .
$$

Hence, for all $s^{*} / 2 \leq s \leq s_{0}$

$$
v(y, s) \leq\left(\frac{T+(M-T) e^{s}}{T}\right)^{\frac{m}{1-m}} k(n, \sigma)^{m} \leq\left(\frac{T+(M-T) e^{s_{0}}}{T}\right)^{\frac{m}{1-m}} k(n, \sigma)^{m} .
$$

It follows that $v$ is uniformly bounded from above. Since $v$ satisfies (49), Proposition 4.4 implies that $v$ has a uniform limit as $s \rightarrow s_{0}$ which is also positive and smooth. By Proposition $4.3 v$ can be extended in a smooth and positive way beyond $s_{0}$, which violates the definition of $s_{0}$. We conclude that $s_{0}=+\infty$. 
Step 3: There is a constant $\kappa_{2}=\left(1+P_{\sigma}(1)(1-m)\right)^{m /(1-m)}>0$ such that for all $s>0$

$$
\min _{\mathbb{S}^{n}} v(y, s) \leq \kappa_{2}
$$

We argue by contradiction. Suppose that there is a time $\bar{s}<\infty$ for which

$$
\min _{\mathbb{S}^{n}} v(y, \bar{s})>\kappa_{2}
$$

This implies

$$
u(x, \bar{t}) \geq\left(T-\bar{t}+P_{\sigma}(1)(1-m)(T-\bar{t})\right)^{1 /(1-m)}\left(\frac{1}{1+|x|^{2}}\right)^{\frac{n+2 \sigma}{2}},
$$

where $\bar{t}=T\left(1-e^{-\bar{s}}\right)<T$. We consider a barrier function

$$
U(x, t)=\left(T-\bar{t}+P_{\sigma}(1)(1-m)(T-t)\right)^{\frac{1}{1-m}}\left(\frac{1}{1+|x|^{2}}\right)^{\frac{n+2 \sigma}{2}},
$$

which satisfies (11). Since $u(x, \bar{t}) \geq U(x, \bar{t})$, by the comparison principle

$$
u(x, \bar{t}) \geq\left(T-\bar{t}+P_{\sigma}(1)(1-m)(T-t)\right)^{\frac{1}{1-m}}\left(\frac{1}{1+|x|^{2}}\right)^{\frac{n+2 \sigma}{2}} .
$$

This contradicts the extinction time $T$ of $u$.

From Steps 1, 2 and 3 we can conclude Proposition 5.1 by taking $\beta_{2}=C \kappa_{2}$ and $\beta_{1}=\kappa_{1} / C$ where $C$ is the constant in Theorem 3.1 for $s_{0}=\infty$.

Now we are in the position to prove Theorem 5.1 Let $J$ be the functional defined as

$$
J(z)=\frac{1}{2} \int_{\mathbb{S}^{n}} z P_{\sigma}(z)-\frac{1}{(1-m)(N+1)} \int_{\mathbb{S}^{n}} z^{N+1} .
$$

Direct computations yield

Lemma 5.1. Let $v(x, s)$ satisfy 49]. Then

$$
\frac{\mathrm{d}}{\mathrm{d} s} J(v(\cdot, s))=-N \int_{\mathbb{S}^{n}} v^{N-1}\left(v_{s}\right)^{2} \leq 0 .
$$

The above lemma indicates that the functional is decreasing in time. The next lemma states that this functional is always nonnegative, and hence $\lim _{s \rightarrow \infty} J(v(\cdot, s))$ exists.

Lemma 5.2. $J(v(\cdot, s)) \geq 0$ for all $s>0$. 
Proof. The proof is similar to that of Lemma 6.1 in [30], which is included here for completeness. We argue by contradiction. Assume that for certain $0<s_{0}<\infty$ one has $J\left(v\left(\cdot, s_{0}\right)\right)<0$. By Lemma 5.1 $J(v(\cdot, s))<0$ for all $s>s_{0}$. Let us consider the quantity

$$
F(s)=\int_{\mathbb{S}^{n}} v^{N+1}(y, s) \mathrm{d} y \geq 0, \quad s \in(0, \infty) .
$$

Then

$$
\begin{aligned}
\frac{N}{N+1} \frac{\mathrm{d}}{\mathrm{d} s} F(s)=\int_{\mathbb{S}^{n}}\left(v^{N}\right)_{s} v & =-2 J(v(\cdot, s))+\frac{N-1}{(1-m)(N+1)} F(s) \\
& \geq \frac{N-1}{(1-m)(N+1)} F(s)
\end{aligned}
$$

for all $s>s_{0}$. Note that $F(s) \neq 0$ for all $s \geq s_{0}$. Otherwise, $v(\cdot, s) \equiv 0$ which is impossible because $J(v(\cdot, s)) \leq J\left(v\left(\cdot, s_{0}\right)\right)<0$. Integrating the above differential inequality, we have

$$
F(s) \geq F\left(s_{0}\right) e^{s-s_{0}} .
$$

It follows that $F(s) \rightarrow \infty$ as $s \rightarrow \infty$. On the other hand, Proposition 5.1 implies that $v$ is uniformly bounded. Consequently, $F(s)$ is bounded. We reach a contradiction.

Proof of Theorem 5.1 It follows from Proposition 5.1 and Proposition 4.4 that for $s>s^{*} / 2$, $v(\cdot, s)$ is compact in $C^{k}\left(\mathbb{S}^{n}\right)$ for any $k$. Let $\bar{v}$ be a limit point of $v(\cdot, s)$ as $s \rightarrow \infty$ in the $C^{2}$ sense. We will show that $\bar{v}$ is a solution of (50) and $\bar{v}$ is the unique limit of $v(\cdot, s)$ as $s \rightarrow \infty$.

Suppose that along a sequence $s_{j} \rightarrow \infty, v\left(\cdot, s_{j}\right) \rightarrow \bar{v}$ in $C^{2}\left(\mathbb{S}^{n}\right)$. Since

$$
\frac{\mathrm{d}}{\mathrm{d} s} J(v(\cdot, s))=-N \int_{\mathbb{S}^{n}} v^{N-1} v_{s}^{2}=-\frac{4 N}{(N+1)^{2}} \int_{\mathbb{S}^{n}}\left|\left(v^{(N+1) / 2}(\cdot, s)\right)_{s}\right|,
$$

we have, by integrating from $s_{j}$ to $s_{j}+\tau$ and using the Cauchy-Schwarz inequality,

$$
\begin{aligned}
& \int_{\mathbb{S}^{n}}\left|v^{\frac{N+1}{2}}\left(\cdot, s_{j}+\tau\right)-v^{\frac{N+1}{2}}\left(\cdot, s_{j}\right)\right|^{2} \\
& \quad \leq \frac{(N+1)^{2} \tau}{4 N}\left(J\left(v\left(\cdot, s_{j}\right)\right)-J\left(v\left(\cdot, s_{j}+\tau\right)\right)\right) .
\end{aligned}
$$

By Lemma 5.1 and Lemma 5.2, $J(v(\cdot, s))$ has a limit as $s \rightarrow \infty$. Hence for each $\tau>0$, $\left\{v\left(\cdot, s_{j}+\tau\right)\right\}_{1}^{\infty}$ is Cauchy in $L^{N+1}$. It follows that $v\left(\cdot, s_{j}+\tau\right) \rightarrow \bar{v}$ in $L^{N+1}$, and in $C^{2}\left(\mathbb{S}^{n}\right)$ uniformly in $\tau$ for $\tau$ in bounded intervals. Thus, for any $\phi \in C^{\infty}\left(\mathbb{S}^{n}\right)$ we have,

$$
\begin{aligned}
\int_{\mathbb{S}^{n}} & \left(v^{N}\left(\cdot, s_{j}+1\right)-v^{N}\left(\cdot, s_{j}\right)\right) \phi \\
\quad= & \int_{0}^{1} \int_{\mathbb{S}^{n}}\left(-P_{\sigma}\left(v\left(y, s_{j}+\tau\right)\right)+\frac{1}{1-m} v^{N}\left(y, s_{j}+\tau\right)\right) \phi \mathrm{d} y \mathrm{~d} \tau .
\end{aligned}
$$


After sending $j \rightarrow \infty$, we obtain

$$
\int_{\mathbb{S}^{n}}\left(-P_{\sigma}(\bar{v})+\frac{1}{1-m} \bar{v}^{N}\right) \phi=0
$$

i.e., $\bar{v}$ solves (50). Finally, it follows from Theorem A.2 that $v(\cdot, s)$ converges to $\bar{v}$ in $C^{3}\left(\mathbb{S}^{n}\right)$.

Proof of Theorem 1.3] By the classification of solutions of (50] in [23] and [57], Theorem 1.3 follows from Theorem 5.1 immediately.

From Theorem 1.3 we see that the extinction profile of $u(x, t)$ is determined by the pair of numbers $\left(\lambda, x_{0}\right)=\left(\lambda\left(u_{0}\right), x_{0}\left(u_{0}\right)\right)$. The next theorem verifies the stability of both the extinction time and the extinction profile.

Theorem 5.2. $T\left(u_{0}\right), \lambda\left(u_{0}\right)$ and $x_{0}\left(u_{0}\right)$ continuously depend on $u_{0}$ in the sense that if $u_{0}$, $\left\{u_{0 ; j}\right\}$ are positive $C^{2}$ functions in $\mathbb{R}^{n},\left(u_{0}^{m}\right)_{0,1},\left(u_{0 ; j}^{m}\right)_{0,1}$ can be extended to positive $C^{2}$ functions near the origin, and $\lim _{j \rightarrow \infty}\left\|u_{0 ; j}^{m}-u_{0}^{m}\right\|_{b}=0$ where $\|\cdot\|_{b}$ is defined by

$$
\|\cdot\|_{b}=\|\cdot\|_{C^{2}\left(B_{2}\right)}+\left\|(\cdot)_{0,1}\right\|_{C^{2}\left(B_{2}\right)},
$$

then

$$
\lim _{j \rightarrow \infty}\left(T\left(u_{0 ; j}\right), \lambda\left(u_{0 ; j}\right), x_{0}\left(u_{0 ; j}\right)\right)=\left(T\left(u_{0}\right), \lambda\left(u_{0}\right), x_{0}\left(u_{0}\right)\right) .
$$

Proof. Given Theorem A.1, Lemma A.1 and Theorem A.2, the proof is identical to the proof of Theorem 1.2 in [30]. We refer to [30] for details.

\section{A Sobolev inequality and a Hardy-Littlewood-Sobolev inequal- ity along a fractional diffusion equation}

As mentioned in the introduction, the results in this section are inspired by [21] and [32].

Proposition 6.1. Assume that $n \geq 2$. If $u$ is a solution of (11) with positive initial data $u_{0} \in C^{2}$ in $\mathbb{R}^{n}$ satisfying that $\left(u_{0}^{m}\right)_{0,1}$ can be extended to a positive $C^{2}$ function near the origin, then

$$
\frac{1}{2} \frac{\mathrm{d}}{\mathrm{d} t} H=\left(\int_{\mathbb{R}^{n}} u^{m+1}\right)^{\frac{2 \sigma}{n}}\left(S_{n, \sigma}\left\|u^{m}\right\|_{\dot{H}^{\sigma}}^{2}-\left\|u^{m}\right\|_{L^{2^{*}(\sigma)}}^{2}\right) \geq 0,
$$

where $H$ is given by (17). 
Proof. It follows from (11) and (13) that

$$
\begin{aligned}
\frac{\mathrm{d}}{\mathrm{d} t} H & =\int_{\mathbb{R}^{n}} 2 u(-\Delta)^{-\sigma} u_{t} \mathrm{~d} x-2 S_{n, \sigma}\left(\int_{\mathbb{R}^{n}} u^{m+1}\right)^{\frac{2 \sigma}{n}} \int_{\mathbb{R}^{n}} u^{m} u_{t} \\
& =-2 \int_{\mathbb{R}^{n}} u^{m+1}+2 S_{n, \sigma}\left(\int_{\mathbb{R}^{n}} u^{m+1}\right)^{\frac{2 \sigma}{n}} \int_{\mathbb{R}^{n}} u^{m}(-\Delta)^{\sigma} u^{m} \\
& =2\left(\int_{\mathbb{R}^{n}} u^{m+1}\right)^{\frac{2 \sigma}{n}}\left(S_{n, \sigma}\left\|u^{m}\right\|_{\dot{H}^{\sigma}}^{2}-\left\|u^{m}\right\|_{L^{2^{*}(\sigma)}}^{2}\right) \geq 0 .
\end{aligned}
$$

Note that the first part of Theorem 5.1, i.e., $v$ defined by (48) is positive and smooth in $\mathbb{S}^{n} \times$ $(0, \infty)$, has been used in the justifications of these equalities.

The next lemma gives an estimate for the extinction time of solutions of (11).

Lemma 6.1. If $u$ is a solution of (11) with positive initial data $u_{0} \in C^{2}$ in $\mathbb{R}^{n}$ satisfying that $\left(u_{0}^{m}\right)_{0,1}$ can be extended to a positive $C^{2}$ function near the origin, then for any $t \in(0, T)$ we have

$$
\left(\frac{4 \sigma(T-t)}{(n+2 \sigma) S_{n, \sigma}}\right)^{\frac{n}{2 \sigma}} \leq \int_{\mathbb{R}^{n}} u^{m+1}(t, x) d x \leq \int_{\mathbb{R}^{n}} u_{0}^{m+1} d x .
$$

Consequently, the extinction time $T$ is bounded by

$$
T \leq \frac{(n+2 \sigma) S_{n, \sigma}}{4 \sigma}\left(\int_{\mathbb{R}^{n}} u_{0}^{m+1} d x\right)^{\frac{2 \sigma}{n}} .
$$

If in addition $n>4 \sigma$, then

$$
T \geq \frac{(n+2 \sigma)}{2 n} \frac{\int_{\mathbb{R}^{n}} u_{0}^{m+1} d x}{\int_{\mathbb{R}^{n}} u_{0}^{m}(-\Delta)^{\sigma} u_{0}^{m}}
$$

and

$$
\begin{aligned}
\int_{\mathbb{R}^{n}} u^{m}(\cdot, t)(-\Delta)^{\sigma} u^{m}(\cdot, t) & \leq \int_{\mathbb{R}^{n}} u_{0}^{m}(-\Delta)^{\sigma} u_{0}^{m}, \\
\int_{\mathbb{R}^{n}} u^{m+1}(\cdot, t) & \geq \int_{\mathbb{R}^{n}} u_{0}^{m+1}-\frac{2 n}{n+2 \sigma} t \int_{\mathbb{R}^{n}} u_{0}^{m}(-\Delta)^{\sigma} u_{0}^{m} .
\end{aligned}
$$

Proof. As in the proof of Lemma 5.2, we define

$$
F(t):=\int_{\mathbb{R}^{n}} u^{m+1}(x, t) \mathrm{d} x,
$$


which is positive in $(0, T)$ and $F(T)=0$. It follows that

$$
\begin{aligned}
F^{\prime}(t) & =(m+1) \int_{\mathbb{R}^{n}} u^{m}(\cdot, t) u_{t}(\cdot, t) \\
& =-(m+1) \int_{\mathbb{R}^{n}} u^{m}(\cdot, t)(-\Delta)^{\sigma} u^{m}(\cdot, t) \leq-\frac{m+1}{S_{n, \sigma}} F(t)^{1-\frac{2 \sigma}{n}}
\end{aligned}
$$

where we have used the Sobolev inequality (13) in the last inequality. This shows the first two inequalities by simple integrations. If in addition $n>4 \sigma$, then

$$
\begin{aligned}
F^{\prime \prime}(t)= & m(m+1) \int_{\mathbb{R}^{n}} u^{m-1}(\cdot, t)\left((-\Delta)^{\sigma} u^{m}(\cdot, t)\right)^{2} \\
& +m(m+1) \int_{\mathbb{R}^{n}} u^{m}(\cdot, t)\left(-\Delta^{\sigma}\right)\left(u^{m-1}(-\Delta)^{\sigma} u^{m}(\cdot, t)\right) \\
= & 2 m(m+1) \int_{\mathbb{R}^{n}} u^{m-1}(\cdot, t)\left((-\Delta)^{\sigma} u^{m}(\cdot, t)\right)^{2} \geq 0,
\end{aligned}
$$

where the condition $n>4 \sigma$ is used to guarantee the $L^{2}$ integrability of $u^{m}(\cdot, t)$ such that we can use Plancherel's theorem in the second equality. Thus, the lower bound of $T$ follows from that $0=F(T) \geq F(t)+F^{\prime}(t)(T-t)$ with sending $t \rightarrow 0$. The last two inequalities follows from the sign of $F^{\prime \prime}$ and simple integrations.

Let

$$
Q:=-\frac{1}{m+1} F^{\prime} F^{\frac{2 \sigma-n}{n}}, E:=-\frac{1}{m+1} F^{\prime} F^{-1}, G\left(t_{1}, t_{2}\right):=\exp \left((m+1) \int_{t_{1}}^{t_{2}} E(s) \mathrm{d} s\right) .
$$

Theorem 6.1. Assume $n>4 \sigma$. For any $u_{0}$ positive and $C^{2}$ in $\mathbb{R}^{n}$ satisfying that $\left(u_{0}^{m}\right)_{0,1}$ can be extended to a positive $C^{2}$ function near the origin, we have

$$
\begin{aligned}
S_{n, \sigma}\left\|u_{0}\right\|_{L^{\frac{2 n}{n+2 \sigma}}}^{2} & -\int_{\mathbb{R}^{n}} u_{0}(-\Delta)^{-\sigma} u_{0} \mathrm{~d} x+4 m S_{n, \sigma} \int_{0}^{T} \mathrm{~d} t \int_{0}^{t} F(s)^{\frac{2 \sigma}{n}} K(s) G(t, s) \mathrm{d} s \\
& =2\left\|u_{0}^{m}\right\|_{L^{2^{*}(\sigma)}}^{\frac{4 \sigma}{n-2 \sigma}}\left(S_{n, \sigma}\left\|u_{0}^{m}\right\|_{\dot{H}^{\sigma}}^{2}-\left\|u_{0}^{m}\right\|_{L^{2^{*}(\sigma)}}^{2}\right) \int_{0}^{T} G(t, 0) \mathrm{d} t,
\end{aligned}
$$

where $u(\cdot, t)$ is the solution of (11) with initial data $u(\cdot, t)=u_{0}, T$ is the extinction time of $u(\cdot, t)$ and $F, E, G, K$ are defined in (52), (53) and (54).

Proof. From the proof of Proposition 6.1 we know that

$$
H^{\prime}(t)=2 F(t)\left(S_{n, \sigma} Q(t)-1\right) .
$$


Hence

$$
\begin{aligned}
H^{\prime \prime}(t) & =2 F^{\prime}(t)\left(S_{n, \sigma} Q(t)-1\right)+2 F(t) S_{n, \sigma} Q^{\prime}(t) \\
& =\frac{F^{\prime}(t)}{F(t)} H^{\prime}(t)+2 F(t) S_{n, \sigma} Q^{\prime}(t) \\
& =-(m+1) E(t) H^{\prime}(t)+2 F(t) S_{n, \sigma} Q^{\prime}(t) .
\end{aligned}
$$

On the other hand,

$$
\begin{aligned}
Q^{\prime}(t) & =\frac{F^{\prime \prime}(t)-\frac{n-2 \sigma}{n} F^{-1}(t)\left(F^{\prime}(t)\right)^{2}}{-(m+1) F(t)^{\frac{n-2 \sigma}{n}}} \\
& =-\frac{2 m}{F(t)^{\frac{n-2 \sigma}{n}}}\left(\int_{\mathbb{R}^{n}} u^{m-1}(\cdot, t)\left((-\Delta)^{\sigma} u^{m}(\cdot, t)\right)^{2}-F^{-1} \int_{\mathbb{R}^{n}} u^{m}(\cdot, t)(-\Delta)^{\sigma} u^{m}(\cdot, t)\right) \\
& =-\frac{2 m}{F(t)^{\frac{n-2 \sigma}{n}}} \int_{\mathbb{R}^{n}} u(\cdot, t)^{m-1}\left|-(-\Delta)^{\sigma} u(\cdot, t)^{m}+E(t) u(\cdot, t)\right|^{2} .
\end{aligned}
$$

Denote

$$
K(t):=\int_{\mathbb{R}^{n}} u(\cdot, t)^{m-1}\left|-(-\Delta)^{\sigma} u(\cdot, t)^{m}+E(t) u(\cdot, t)\right|^{2} .
$$

Then

$$
H^{\prime \prime}(t)=-(m+1) E(t) H^{\prime}(t)-4 m F^{\frac{2 \sigma}{n}}(t) S_{n, \sigma} K(t) .
$$

Multiplying $G(0, s)$ and integrating from 0 to $t$, we have

$$
H^{\prime}(t) G(0, t)-H^{\prime}(0) G(0,0)=\int_{0}^{t}\left(H^{\prime} G\right)^{\prime}(s) \mathrm{d} s=-4 m S_{n, \sigma} \int_{0}^{t} F(s)^{\frac{2 \sigma}{n}} K(s) G(0, s) \mathrm{d} s .
$$

Dividing $G(0, t)$ and integrating from 0 to $T$, we obtain

$$
0-H(0)=H^{\prime}(0) \int_{0}^{T} G(t, 0) \mathrm{d} t-4 m S_{n, \sigma} \int_{0}^{T} \mathrm{~d} t \int_{0}^{t} F(s)^{\frac{2 \sigma}{n}} K(s) G(t, s) \mathrm{d} s,
$$

which finishes the proof.

The drawback of the above Theorem is that the extra terms are not explicit. Fortunately, we can use simple estimates to reach Theorem 1.4

Proof of Theorem 1.4 We first assume that $w=u_{0}^{m}$ where $u_{0} \in C^{2}\left(\mathbb{R}^{n}\right)$ is positive and satisfies that $\left(u_{0}^{m}\right)_{0,1}$ can be extended to a positive $C^{2}$ function near the origin. By Lemma 6.1 ,

$$
(m+1) E(s) \geq(m+1) S_{n, \sigma}^{-1}\left(\int_{\mathbb{R}^{n}} u(\cdot, s)^{m+1}\right)^{-2 \sigma / n} \geq(m+1) S_{n, \sigma}^{-1}\left(\int_{\mathbb{R}^{n}} u_{0}^{m+1}\right)^{-2 \sigma / n}=: b .
$$


By Lemma 6.1 again, we have $b T \leq \frac{n}{2 \sigma}$. Therefore,

$$
\begin{aligned}
\int_{0}^{T} G(t, 0) \mathrm{d} t \leq \int_{0}^{T} e^{-b t} \mathrm{~d} t & =\frac{1-e^{-b T}}{b} \\
& \leq \frac{1-e^{-\frac{n}{2 \sigma}}}{m+1} S_{n, \sigma}\left(\int_{\mathbb{R}^{n}} u_{0}^{m+1}\right)^{2 \sigma / n}
\end{aligned}
$$

Hence (18) holds for $w=u_{0}^{m}$ where $u_{0} \in C^{2}\left(\mathbb{R}^{n}\right)$ is positive and satisfies that $\left(u_{0}^{m}\right)_{0,1}$ can be extended to a positive $C^{2}$ function near the origin.

For any nonnegative $u \in C_{c}^{\infty}\left(\mathbb{R}^{n}\right)$, we consider $w_{\varepsilon}=u+\varepsilon\left(\frac{2}{1+|x|^{2}}\right)^{\frac{n-2 \sigma}{2}}$ with $\varepsilon>0$. Then (18) holds for $w_{\varepsilon}$. By sending $\varepsilon \rightarrow 0$, we have (18) for $u$. Finally, Theorem 1.4 follows from a density argument.

\section{A Uniqueness theorem for negative gradient flow involving nonlo- cal operator}

In this appendix, we provide a uniqueness theorem for fractional Yamabe flows, which is analog to L. Simon's uniqueness Theorem in [72]. The proofs are essentially the same and we will just sketch them in our setting. Denote $H^{\sigma}\left(\mathbb{S}^{n}\right)$ as the closure of $C^{\infty}\left(\mathbb{S}^{n}\right)$ under the norm

$$
\|v\|_{H^{\sigma}\left(\mathbb{S}^{n}\right)}=\int_{\mathbb{S}^{n}} v P_{\sigma}(v) .
$$

Let $\alpha \in(0,1)$ such that $2 \sigma+\alpha$ is not an integer. Let $J$ be the functional defined as

$$
J(v)=\frac{1}{2} \int_{\mathbb{S}^{n}} v P_{\sigma}(v)-\frac{1}{(1-m)(N+1)} \int_{\mathbb{S}^{n}} v^{N+1}, \quad v \in H^{\sigma}\left(\mathbb{S}^{n}\right) .
$$

Then

$$
\nabla J(v)=P_{\sigma}(v)-\frac{1}{1-m} v^{N} .
$$

Let $\bar{v}$ be such that $\nabla J(\bar{v})=0$.

Theorem A.1. There exist $\theta \in(0,1 / 2)$ and $r_{0}>0$ such that for any $v \in C^{2 \sigma+\alpha}\left(\mathbb{S}^{n}\right)$ with $\|v-\bar{v}\|_{C^{2 \sigma+\alpha}}<r_{0}$,

$$
\|\nabla J(v)\|_{L^{2}\left(\mathbb{S}^{n}\right)} \geq|J(v)-J(\bar{v})|^{1-\theta} .
$$

Proof. Since we have Schauder estimates (see, e.g., [51]) and $L^{2}$ estimates (which is free from equivalence of definitions of fractional Sobolev spaces on $\mathbb{S}^{n}$ ) for $P_{\sigma}$, the proof is identical to that of Theorem 3 in [72]. 
Let $v(x, s)$ and $\bar{v}$ be as in Section 5. Then direction computations and uniform bounds of $v(x, s)$ yield the following lemma

Lemma A.1. There exist two constants $c_{0}$ and $T_{0}$ such that for any $t>T_{0}$ we have

$$
-\frac{\mathrm{d}}{\mathrm{d} s} J(v(\cdot, s)) \geq c_{0}\left\|v_{s}\right\|_{L^{2}\left(\mathbb{S}^{n}\right)}\|\nabla J(v(\cdot, s))\|_{L^{2}\left(\mathbb{S}^{n}\right)} .
$$

Theorem A.2.

$$
\lim _{s \rightarrow \infty}\|v(\cdot, s)-\bar{v}\|_{C^{l}\left(\mathbb{S}^{n}\right)}=0
$$

for any positive integer $l$.

Proof. First we can prove that $v(\cdot, t)$ converges to $\bar{v}$ in $C^{2 \sigma+\alpha}\left(\mathbb{S}^{n}\right)$, using the same methods as the proof of Proposition 21 in [1] or the proof of Theorem 1 in [43]. Then Theorem A.2 follows from the uniform $C^{l+1}$ bound of $v(x, s)$.

Similarly if

$$
S(z)=\frac{\int_{\mathbb{S}^{n}} z P_{\sigma}(z)}{\left(\int_{\mathbb{S}^{n}} z^{N+1}\right)^{\frac{2}{N+1}}}, \quad z \in H^{\sigma}\left(\mathbb{S}^{n}\right)
$$

then

$$
\nabla S(z)=2\left(\int_{\mathbb{S}^{n}} z^{N+1}\right)^{-\frac{2}{N+1}}\left(P_{\sigma}(z)-\frac{\int_{\mathbb{S}^{n}} z P_{\sigma}(z)}{\int_{\mathbb{S}^{n}} z^{N+1}} z^{N}\right) .
$$

Let $v(x, t)$ and $v_{\infty}$ be as in Theorem 1.1 Note that $\nabla S\left(v_{\infty}\right)=0$.

Theorem A.3. There exist $\theta \in(0,1 / 2)$ and $r_{0}>0$ such that for any $\left\|v-v_{\infty}\right\|_{C^{2 \sigma+\alpha}}<r_{0}$,

$$
\|\nabla S(v)\|_{L^{2}\left(\mathbb{S}^{n}\right)} \geq\left|S(v)-S\left(v_{\infty}\right)\right|^{1-\theta} .
$$

Lemma A.2. There exist two constants $c_{0}$ and $T_{0}$ such that for any $t>T_{0}$ we have

$$
-\frac{\mathrm{d}}{\mathrm{d} t} S(v(\cdot, t)) \geq c_{0}\left\|v_{t}\right\|_{L^{2}\left(\mathbb{S}^{n}\right)}\|\nabla S(v(\cdot, t))\|_{L^{2}\left(\mathbb{S}^{n}\right)} .
$$

\section{Theorem A.4.}

$$
\lim _{t \rightarrow \infty}\left\|v(\cdot, t)-v_{\infty}\right\|_{C^{l}\left(\mathbb{S}^{n}\right)}=0
$$

for any positive integer $l$. 


\section{B Some interpolation inequalities}

Lemma B.1. Suppose that $0<\alpha<\min (1,2 \sigma)$ and $2 \sigma+\alpha$ is not an integer. There exists $a$ constant $C>0$ depending only on $n$ and $\sigma$ such that for any $\varepsilon>0$ and $u \in \mathcal{C}^{2 \sigma+\alpha, 1+\frac{\alpha}{2 \sigma}}\left(Q_{T}\right)$, we have

$$
\begin{aligned}
\left|u_{t}\right|_{0 ; Q_{T}} & \leq \varepsilon\left[u_{t}\right]_{\alpha, \frac{\alpha}{2 \sigma} ; Q_{T}}+C \varepsilon^{-2 \sigma / \alpha}|u|_{0 ; Q_{T}}, \\
\left|(-\Delta)^{\sigma} u\right|_{0 ; Q_{T}} & \leq \varepsilon[u]_{2 \sigma+\alpha, 1+\frac{\alpha}{2 \sigma} ; Q_{T}}+C \varepsilon^{-2 \sigma / \alpha}|u|_{0 ; Q_{T}}, \\
{[u]_{\alpha, \frac{\alpha}{2 \sigma} ; Q_{T}} } & \leq \varepsilon[u]_{2 \sigma+\alpha, 1+\frac{\alpha}{2 \sigma} ; Q_{T}}+C \varepsilon^{-\alpha /(2 \sigma)}|u|_{0 ; Q_{T}} .
\end{aligned}
$$

If $\sigma>\frac{1}{2}$, then

$$
\left[\nabla_{x} u\right]_{\alpha, \frac{\alpha}{2 \sigma} ; Q_{T}} \leq \varepsilon[u]_{2 \sigma+\alpha, 1+\frac{\alpha}{2 \sigma} ; Q_{T}}+C \varepsilon^{-(1+\alpha) /(2 \sigma-1)}|u|_{0 ; Q_{T}} .
$$

Proof. By the fractional parabolic dilations of the form $u(x, t) \rightarrow u\left(R x, R^{2 \sigma} t\right)$, we only need to show the case $\varepsilon=1$ and $T=2$. Take $X=(x, t) \in Q_{T}$ and we have, for some $\theta \in(-1,1)$,

$$
\begin{aligned}
\left|u_{t}(X)\right| & \leq\left|u_{t}(X)-(u(x, t \pm 1)-u(x, t))\right|+2|u|_{0 ; Q_{T}} \\
& =\left|u_{t}(X)-u_{t}(x, t+\theta)\right|+2|u|_{0 ; Q_{T}} \leq\left[u_{t}\right]_{\alpha, \frac{\alpha}{2 \sigma} ; Q_{T}}+2|u|_{0 ; Q_{T}} .
\end{aligned}
$$

This finishes the proof of (55). For (56) and (57), we first recall (see, e.g., [73]) that

$$
|w|_{2 \sigma+\alpha ; \mathbb{R}^{n}} \leq C\left(|w|_{0 ; \mathbb{R}^{n}}+\left|(-\Delta)^{\sigma} w\right|_{\alpha ; \mathbb{R}^{n}}\right) \quad \text { for all } w \in C^{2 \sigma+\alpha}\left(\mathbb{R}^{n}\right) .
$$

Hence,

$$
\begin{aligned}
\left|(-\Delta)^{\sigma} u(x, t)\right| & \leq C\left(|u(\cdot, t)|_{0 ; \mathbb{R}^{n}}+|u(\cdot, t)|_{C^{2 \sigma+\alpha}\left(\mathbb{R}^{n}\right)}\right) \\
& \leq C\left(|u|_{0 ; Q_{T}}+\left[(-\Delta)^{\sigma} u\right]_{\alpha, \frac{\alpha}{2 \sigma} ; Q_{T}}\right) \leq C\left([u]_{2 \sigma+\alpha, 1+\frac{\alpha}{2 \sigma} ; Q_{T}}+|u|_{0 ; Q_{T}}\right),
\end{aligned}
$$

and

$$
\begin{aligned}
{[u]_{\alpha, \frac{\alpha}{2 \sigma} ; Q_{T}} } & \leq \sup _{t_{1} \neq t_{2}, x} \frac{\left|u\left(x, t_{1}\right)-u\left(x, t_{2}\right)\right|}{\left|t_{1}-t_{2}\right|^{\frac{\alpha}{2 \sigma}}}+\sup _{x_{1} \neq x_{2}, t} \frac{\left|u\left(x_{1}, t\right)-u\left(x_{2}, t\right)\right|}{\left|x_{1}-x_{2}\right|^{\alpha}} \\
& \leq C\left(|u|_{0 ; Q_{T}}+\left|u_{t}\right|_{0 ; Q_{T}}+\sup _{t}|u(\cdot, t)|_{2 \sigma+\alpha ; \mathbb{R}^{n}}\right) \\
& \leq C\left([u]_{2 \sigma+\alpha, 1+\frac{\alpha}{2 \sigma} ; Q_{T}}+|u|_{0 ; Q_{T}}\right) .
\end{aligned}
$$

Finally, for $\sigma>\frac{1}{2}$ we notice that by the same methods as above,

$$
\sup _{t, x_{1} \neq x_{2}} \frac{\left|\nabla_{x} u\left(x_{1}, t\right)-\nabla_{x} u\left(x_{2}, t\right)\right|}{\left|x_{1}-x_{2}\right|^{\alpha}} \leq C\left([u]_{2 \sigma+\alpha, 1+\frac{\alpha}{2 \sigma} ; Q_{T}}+|u|_{0 ; Q_{T}}\right) .
$$


Thus, to prove (58), we only need to show

$$
\sup _{s \neq t, x} \frac{\left|\nabla_{x} u(x, s)-\nabla_{x} u(x, t)\right|}{|s-t|^{\frac{\alpha}{2 \sigma}}} \leq C\left([u]_{2 \sigma+\alpha, 1+\frac{\alpha}{2 \sigma} ; Q_{T}}+|u|_{0 ; Q_{T}}\right) .
$$

Fix any $x_{0} \in \mathbb{R}^{n}$. Let $w(x, t)=(-\Delta)^{\sigma} u(x, t)$ and $\eta(x)$ be a smooth cut-off function supported in $B_{2}\left(x_{0}\right) \in \mathbb{R}^{n}$ and equal to 1 in $B_{1}\left(x_{0}\right)$. Let

$$
u_{0}(x, t)=(-\Delta)^{-\sigma}(\eta w)=\int_{\mathbb{R}^{n}} \frac{\eta(y) w(y, t)}{|x-y|^{n-2 \sigma}} \mathrm{d} y .
$$

For convenience we have omitted some positive constant as in (16). Then

$$
(-\Delta)^{\sigma}\left(u_{0}(x, t)-u(x, t)-u_{0}(x, s)+u(x, s)\right)=0 \quad \text { in } B_{1}\left(x_{0}\right),
$$

which implies, for $0<|t-s| \leq 1$,

$$
\begin{aligned}
& \left|\nabla_{x} u_{0}\left(x_{0}, t\right)-\nabla_{x} u\left(x_{0}, t\right)-\nabla_{x} u_{0}\left(x_{0}, s\right)+\nabla_{x} u\left(x_{0}, s\right)\right| \\
& \leq C\left|u_{0}(x, t)-u(x, t)-u_{0}(x, s)+u(x, s)\right|_{L^{\infty}\left(\mathbb{R}^{n}\right)} \\
& \leq C\left(\left|u_{t}\right|_{0 ; Q_{T}}+[u]_{2 \sigma+\alpha, 1+\frac{\alpha}{2 \sigma} ; Q_{T}}\right)|t-s|^{\frac{\alpha}{2 \sigma}} .
\end{aligned}
$$

Since $\sigma>1 / 2$ and

$$
\nabla_{x} u_{0}\left(x_{0}, t\right)=(2 \sigma-n) \int_{\mathbb{R}^{n}} \frac{\left(x_{0}-y\right) \eta(y) w(y, t)}{\left|x_{0}-y\right|^{n+2-2 \sigma}} \mathrm{d} y,
$$

we have

$$
\left|\nabla_{x} u_{0}\left(x_{0}, t\right)-\nabla_{x} u_{0}\left(x_{0}, s\right)\right| \leq C[u]_{2 \sigma+\alpha, 1+\frac{\alpha}{2 \sigma} ; Q_{T}}|t-s|^{\frac{\alpha}{2 \sigma}} .
$$

Together with (55), we have

$$
\sup _{s \neq t, x} \frac{\left|\nabla_{x} u(x, s)-\nabla_{x} u(x, t)\right|}{|s-t|^{\frac{\alpha}{2 \sigma}}} \leq C\left([u]_{2 \sigma+\alpha, 1+\frac{\alpha}{2 \sigma} ; Q_{T}}+|u|_{0 ; Q_{T}}\right) .
$$

This finishes the proof of (58).

Lemma B.2. Suppose that $0<\alpha<\min (1,2 \sigma)$ and $2 \sigma+\alpha$ is not an integer. For any small $\varepsilon>0$, there exists a constant $C(\varepsilon)>0$ depending only on $n, \sigma$ and $\varepsilon$ such that for any $v \in \mathcal{C}^{2 \sigma+\alpha, 1+\frac{\alpha}{2 \sigma}}\left(\mathcal{Q}_{T}\right)$, we have

$$
\begin{aligned}
\left|v_{t}\right|_{0 ; \mathcal{Q}_{T}} & \leq \varepsilon\left[v_{t}\right]_{\alpha, \frac{\alpha}{2 \sigma} ; \mathcal{Q}_{T}}+C(\varepsilon)|v|_{0 ; \mathcal{Q}_{T}} \\
\left|P_{\sigma} v\right|_{0 ; \mathcal{Q}_{T}} & \leq \varepsilon[v]_{2 \sigma+\alpha, 1+\frac{\alpha}{2 \sigma} ; \mathcal{Q}_{T}}+C(\varepsilon)|v|_{0 ; \mathcal{Q}_{T}}, \\
{[v]_{\alpha, \frac{\alpha}{2 \sigma} ; \mathcal{Q}_{T}} } & \leq \varepsilon[v]_{2 \sigma+\alpha, 1+\frac{\alpha}{2 \sigma} ; \mathcal{Q}_{T}}+C(\varepsilon)|v|_{0 ; \mathcal{Q}_{T}}
\end{aligned}
$$


Proof. Using stereographic projections, (9) and noticing that $|x-y| \geq C_{n}|F(x)-F(y)|$, the above inequalities follows from LemmaB.1

Lemma B.3. Suppose that $0<\alpha<\min (1,2 \sigma)$ and $2 \sigma+\alpha$ is not an integer. Let $u \in$ $\mathcal{C}^{2 \sigma+\alpha, 1+\frac{\alpha}{2 \sigma}}\left(Q_{1}\right)$ and $\eta \in C_{c}^{2}\left(\mathbb{R}^{n+1}\right)$, then for any $\varepsilon>0$, there exists $C(\varepsilon)>0$ depending only on $\alpha, \sigma, n, \varepsilon$ and $\|\eta\|_{C^{2}\left(\mathbb{R}^{n+1}\right)}$ such that

$$
[\langle u, \eta\rangle]_{\alpha, \frac{\alpha}{2 \sigma} ; Q_{1}} \leq \varepsilon[u]_{2 \sigma+\alpha, 1+\frac{\alpha}{2 \sigma} ; Q_{1}}+C(\varepsilon)|u|_{0, Q_{1}} .
$$

Proof. We denote $C$ as various constants depending only on $n, \sigma, \alpha,\|\eta\|_{C^{2}\left(\mathbb{R}^{n+1}\right)}$, and $C(\varepsilon)$ as various constants depending only on $n, \sigma, \alpha,\|\eta\|_{C^{2}\left(\mathbb{R}^{n+1}\right)}$ and $\varepsilon$. Recall that $\langle u, \eta\rangle$ is defined in (35). For any $(x, t) \in Q_{1}$,

$$
\begin{aligned}
|\langle u, \eta\rangle(x, t)| \leq & c(n, \sigma) \int_{\mathbb{R}^{n} \backslash B_{1}(x)} \frac{|u(x, t)-u(y, t)||\eta(x, t)-\eta(y, t)|}{|x-y|^{n+2 \sigma}} \mathrm{d} y \\
& +c(n, \sigma) \int_{B_{1}(x)} \frac{|u(x, t)-u(y, t)||\eta(x, t)-\eta(y, t)|}{|x-y|^{n+2 \sigma}} \mathrm{d} y \\
\leq & C|u|_{0, Q_{1}}+C[u(\cdot, t)]_{\sigma, \mathbb{R}^{n}} \leq \varepsilon[u]_{2 \sigma+\alpha, 1+\frac{\alpha}{2 \sigma} ; Q_{1}}+C(\varepsilon)|u|_{0, Q_{1}} .
\end{aligned}
$$

Fix any $X_{1}=\left(x_{1}, t_{1}\right), X_{2}=\left(x_{2}, t_{2}\right) \in Q_{1}, X_{1} \neq X_{2}$. For convenience, we write $\rho=$ $\rho\left(X_{1}, X_{2}\right)$ and $u^{z}(x, t)=u(x, t)-u(x+z, t)$. We may also suppose that $\rho \leq 1$.

$$
\begin{aligned}
& \left|\langle u, \eta\rangle\left(x_{1}, t_{1}\right)-\langle u, \eta\rangle\left(x_{2}, t_{2}\right)\right| \\
& \leq\left|\int_{|z| \leq \rho} \frac{\left(u^{z}\left(x_{1}, t_{1}\right)-u^{z}\left(x_{2}, t_{2}\right)\right) \eta^{z}\left(x_{1}, t_{1}\right)}{|z|^{n+2 \sigma}} \mathrm{d} z\right| \\
& +\left|\int_{|z| \leq \rho} \frac{\left(\eta^{z}\left(x_{1}, t_{1}\right)-\eta^{z}\left(x_{2}, t_{2}\right)\right) u^{z}\left(x_{2}, t_{2}\right)}{|z|^{n+2 \sigma}} \mathrm{d} z\right| \\
& +\left|\int_{|z| \geq \rho} \frac{\left(u^{z}\left(x_{1}, t_{1}\right)-u^{z}\left(x_{2}, t_{2}\right)\right) \eta^{z}\left(x_{1}, t_{1}\right)}{|z|^{n+2 \sigma}} \mathrm{d} z\right| \\
& +\left|\int_{|z| \geq \rho} \frac{\left(\eta^{z}\left(x_{1}, t_{1}\right)-\eta^{z}\left(x_{2}, t_{2}\right)\right) u^{z}\left(x_{2}, t_{2}\right)}{|z|^{n+2 \sigma}} \mathrm{d} z\right| \\
& :=I_{1}+I_{2}+I_{3}+I_{4}
\end{aligned}
$$

For $I_{1}$ and $I_{2}$, we first consider that $2 \sigma+\alpha<1$. Then by change of variable,

$$
I_{1}+I_{2} \leq C \max _{i=1,2}\left[u\left(\cdot, t_{i}\right)\right]_{\alpha+\sigma ; \mathbb{R}^{n}} \int_{|z| \leq \rho}|z|^{\alpha+\sigma+1-n-2 \sigma} \mathrm{d} z \leq C \max _{i=1,2}\left[u\left(\cdot, t_{i}\right)\right]_{\alpha+\sigma ; \mathbb{R}^{n}} \rho^{1+\alpha-\sigma} .
$$


If $1<\alpha+2 \sigma<2$, we have

$$
I_{1}+I_{2} \leq C \max _{i=1,2}\left[u\left(\cdot, t_{i}\right)\right]_{\alpha+2 \sigma-1 ; \mathbb{R}^{n}} \int_{|z| \leq \rho}|z|^{\alpha+2 \sigma-n-2 \sigma} \mathrm{d} z \leq C \max _{i=1,2}\left[u\left(\cdot, t_{i}\right)\right]_{\alpha+2 \sigma-1 ; \mathbb{R}^{n}} \rho^{\alpha} .
$$

If $2 \sigma+\alpha>2$, then

$$
\begin{aligned}
I_{1} & \leq\left|\int_{|z| \leq \rho} \frac{\left(u^{z}\left(x_{1}, t_{1}\right)+\nabla_{x} u\left(x_{1}, t_{1}\right) z-u^{z}\left(x_{2}, t_{2}\right)-\nabla_{x} u\left(x_{2}, t_{2}\right) z\right) \eta^{z}\left(x_{1}, t_{1}\right)}{|z|^{n+2 \sigma}} \mathrm{d} z\right| \\
& +\left|\int_{|z| \leq \rho} \frac{\left(\nabla_{x} u\left(x_{1}, t_{1}\right) z-\nabla_{x} u\left(x_{2}, t_{2}\right) z\right) \eta^{z}\left(x_{1}, t_{1}\right)}{|z|^{n+2 \sigma}} \mathrm{d} z\right| \\
& \leq C \sup _{Q_{1}}\left|\nabla_{x}^{2} u\right| \int_{|z| \leq \rho}|z|^{3-n-2 \sigma} \mathrm{d} z+C\left[\nabla_{x} u\right]_{\alpha, \frac{\alpha}{2 \sigma} ; Q_{T}} \rho^{\alpha} \int_{|z| \leq \rho}|z|^{2-n-2 \sigma} \mathrm{d} z \\
& \leq \rho^{\alpha}\left(\varepsilon[u]_{2 \sigma+\alpha, 1+\frac{\alpha}{2 \sigma} ; Q_{T}}+C(\varepsilon)|u|_{0 ; Q_{T}}\right) .
\end{aligned}
$$

Similarly,

$$
\begin{aligned}
I_{2} & \leq C\left|\nabla_{x} u\right|_{0 ; Q_{1}} \int_{|z| \leq \rho}|z|^{3-n-2 \sigma} \mathrm{d} z+C\left|\nabla_{x} u\right|_{0 ; Q_{1}} \rho^{\alpha} \int_{|z| \leq \rho}|z|^{2-n-2 \sigma} \mathrm{d} z \\
& \leq \rho^{\alpha}\left(\varepsilon[u]_{2 \sigma+\alpha, 1+\frac{\alpha}{2 \sigma} ; Q_{T}}+C(\varepsilon)|u|_{0 ; Q_{T}}\right) .
\end{aligned}
$$

For $I_{3}$ and $I_{4}$ we first consider that $\sigma \leq \frac{1}{2}$. Choose an $\alpha^{\prime}>\alpha$ but sufficiently close to $\alpha$ such that $\alpha^{\prime}<\min (1,2 \sigma)$, then

$$
\begin{gathered}
I_{3} \leq[u]_{\alpha^{\prime}, \frac{\alpha^{\prime}}{2 \sigma} ; Q_{1}} \rho^{\alpha^{\prime}} C \int_{|z| \geq \rho}|z|^{2 \sigma+\alpha-\alpha^{\prime}-n-2 \sigma} \mathrm{d} z \leq C[u]_{\alpha^{\prime}, \frac{\alpha^{\prime}}{2 \sigma} ; Q_{1}} \rho^{\alpha}, \\
I_{4} \leq C \rho^{\alpha^{\prime}}\left[u\left(\cdot, t_{2}\right)\right]_{2 \sigma+\alpha-\alpha^{\prime} ; \mathbb{R}^{n}} \int_{|z| \geq \rho}|z|^{2 \sigma+\alpha-\alpha^{\prime}-n-2 \sigma} \mathrm{d} z \leq C\left[u\left(\cdot, t_{2}\right)\right]_{2 \sigma+\alpha-\alpha^{\prime} ; \mathbb{R}^{n}} \rho^{\alpha} .
\end{gathered}
$$

If $\sigma>\frac{1}{2}$ and $2 \sigma+\alpha<2$, then

$$
\begin{gathered}
I_{3} \leq[u]_{2 \sigma+\alpha-1, \frac{2 \sigma+\alpha-1}{2 \sigma} ; Q_{1}} \rho^{2 \sigma+\alpha-1} C \int_{|z| \geq \rho}|z|^{1-n-2 \sigma} \mathrm{d} z \leq C[u]_{2 \sigma+\alpha-1, \frac{2 \sigma+\alpha-1}{2 \sigma} ; Q_{1}} \rho^{\alpha}, \\
I_{4} \leq C \rho^{2 \sigma+\alpha-1}\left|\nabla u\left(\cdot, t_{2}\right)\right|_{0 ; \mathbb{R}^{n}} \int_{|z| \geq \rho}|z|^{1-n-2 \sigma} \mathrm{d} z \leq C\left|\nabla u\left(\cdot, t_{2}\right)\right|_{0 ; \mathbb{R}^{n}} \rho^{\alpha} .
\end{gathered}
$$

If $2 \sigma+\alpha>2$, then for $\rho \leq|z| \leq 1$, we have

$\left|u^{z}\left(x_{1}, t_{1}\right)-u^{z}\left(x_{2}\right)\right| \leq\left|\nabla_{x}^{2} u\right|_{0 ; Q_{1}}\left|x_{1}-x_{2}\right||z|+\left|u_{t}\right|_{0 ; Q_{1}}\left|t_{1}-t_{2}\right| \leq\left|\nabla_{x}^{2} u\right|_{0 ; Q_{1}} \rho|z|+\left|u_{t}\right|_{0 ; Q_{1}} \mid \rho^{2 \sigma}$. 
Hence,

$$
\begin{aligned}
I_{3} & \leq\left|\int_{1 \geq|z| \geq \rho} \frac{\left(u^{z}\left(x_{1}, t_{1}\right)-u^{z}\left(x_{2}, t_{2}\right)\right) \eta^{z}\left(x_{1}, t_{1}\right)}{|z|^{n+2 \sigma}} \mathrm{d} z\right| \\
& +\left|\int_{|z| \geq 1} \frac{\left(u^{z}\left(x_{1}, t_{1}\right)-u^{z}\left(x_{2}, t_{2}\right)\right) \eta^{z}\left(x_{1}, t_{1}\right)}{|z|^{n+2 \sigma}} \mathrm{d} z\right| \\
& \leq C\left|\nabla_{x}^{2} u\right|_{0 ; Q_{1}} \rho \int_{1 \geq|z| \geq \rho}|z|^{2-n-2 \sigma} \mathrm{d} z+C\left|u_{t}\right|_{0 ; Q_{1}} \rho^{2 \sigma} \int_{1 \geq|z| \geq \rho}|z|^{1-n-2 \sigma} \\
& +[u]_{\alpha, \frac{\alpha}{2 \sigma} ; Q_{1}} \rho^{\alpha} \int_{|z| \geq 1}|z|^{-n-2 \sigma} \mathrm{d} z \\
\leq & C\left(\left|\nabla_{x}^{2} u\right|_{0 ; Q_{1}}+\left|u_{t}\right|_{0 ; Q_{1}}+[u]_{\alpha, \frac{\alpha}{2 \sigma} ; Q_{1}}\right) \rho^{\alpha} .
\end{aligned}
$$

Similarly, for $I_{4}$ we have

$$
I_{4} \leq C\left|\nabla_{x} u\right|_{0 ; Q_{1}} \rho^{\alpha} .
$$

Combining these and applying some interpolation inequalities in Lemma B.1, we reach (62).

\section{References}

[1] B. Andrews, Monotone quantities and unique limits for evolving convex hypersurfaces, Internat. Math. Res. Notices 1997, no. 20, 1001-1031.

[2] I. Athanasopoulos and L. Caffarelli, Continuity of the temperature in boundary heat control problems, Adv. Math. 224 (2010), 293-315.

[3] T. Aubin, Problèmes isopérimétriques et espaces de Sobolev, J. Differential Geom. 11 (1976), no. 4, 573-598.

[4] P. Baird, A. Fardoun and R. Regbaoui, Q-curvature flow on 4-manifolds, Calc. Var. Partial Differential Equations 27 (2006), no. 1, 75-104.

[5] W. Beckner, Sharp Sobolev inequalities on the sphere and the Moser-Trudinger inequality, Ann. of Math. (2) 138 (1993), no. 1, 213-242.

[6] G. Bianchi and H. Egnell, A note on the Sobolev inequality, J. Funct. Anal. 100 (1991), no. 1, 18-24.

[7] M. Birkner, J. A. López-Mimbela and A. Wakolbinger, Comparison results and steady states for the Fujita equation with fractional Laplacian, Ann. Inst. H. Poincaré Anal. Non Linéaire 22 (2005), no. 1, 83-97. 
[8] A. Blanchet, M. Bonforte, J. Dolbeault, G. Grillo and J.L. Vázquez, Asymptotics of the fast diffusion equation via entropy estimates, Arch. Ration. Mech. Anal. 191 (2009), no. $2,347-385$.

[9] M. Bonforte, J. Dolbeault, G. Grillo and J.L. Vázquez, Sharp rates of decay of solutions to the nonlinear fast diffusion equation via functional inequalities, Proc. Natl. Acad. Sci. USA 107 (2010), no. 38, 16459-16464.

[10] T.P. Branson, Sharp inequalities, the functional determinant, and the complementary series, Trans. Amer. Math. Soc. 347 (1995), 367-3742.

[11] S. Brendle, A generalization of the Yamabe flow for manifolds with boundary, Asian J. Math. 6 (2002), no. 4, 625-644.

[12] S. Brendle, Global existence and convergence for a higher order flow in conformal geometry, Ann. of Math. (2) 158 (2003), no. 1, 323-343.

[13] S. Brendle, Convergence of the Yamabe flow for arbitrary initial energy, J. Differential Geom. 69 (2005), 217-278.

[14] S. Brendle, Convergence of the $Q$-curvature flow on $\mathbb{S}^{4}$, Adv. Math. 205 (2006), no. 1, $1-32$.

[15] S. Brendle, Convergence of the Yamabe flow in dimension 6 and higher, Invent. Math. 170 (2007), no. 3, 541-576.

[16] H. Brezis and E. Lieb, Sobolev inequalities with remainder terms, J. Funct. Anal. 62 (1985), no. 1, 73-86.

[17] H. Brezis and L. Nirenberg, Positive solutions of nonlinear elliptic equations involving critical Sobolev exponents, Comm. Pure Appl. Math. 36 (1983), no. 4, 437-477.

[18] X. Cabre and Y. Sire, Nonlinear equations for fractional Laplacians I: Regularity, maximum principles, and Hamiltonian estimates, arXiv:1012.0867.

[19] L. Caffarelli and L. Silvestre, An extension problem related to the fractional Laplacian, Comm. Partial. Diff. Equ. 32 (2007), 1245-1260.

[20] L. Caffarelli and J.L. Vazquez, Nonlinear porous medium flow with fractional potential pressure, Arch. Rational Mech. Anal. 202 (2011), no. 2, 537-565.

[21] E. Carlen, J. arrillo and M. Loss, Hardy-Littlewood-Sobolev inequalities via fast diffusion flows, Proc. Natl. Acad. Sci. USA 107 (2010), no. 46, 19696-19701. 
[22] S.-Y. Chang and M. González, Fractional Laplacian in conformal geometry, Adv. Math. 226 (2011), 1410-1432.

[23] W. Chen, C. $\mathrm{Li}$ and B. Ou, Classification of solutions for an integral equation, Comm. Pure Appl. Math. 59 (2006), 330-343.

[24] P. Cherrier, Problèmes de Neumann non linéaires sur les variétés riemanniennes, J. Funct. Anal. 57 (1984), no. 2, 154-206.

[25] B. Chow, The Yamabe flow on locally conformally flat manifolds with positive Ricci curvature, Comm. Pure Appl. Math. 45 (1992), 1003-1014.

[26] A. Cianchi, N. Fusco, F. Maggi and A. Pratelli, The sharp Sobolev inequality in quantitative form, J. Eur. Math. Soc. (JEMS) 11 (2009), no. 5, 1105-1139.

[27] P. Daskalopoulos and N. Sesum, On the extinction profile of solutions to fast diffusion, J. Reine Angew. Math. 622 (2008), 95-119.

[28] A. de Pablo, F. Quiros, A. Rodriguez and J. Vazquez, A fractional porous medium equation, Adv. Math. 226 (2011), no. 2, 1378-1409.

[29] A. de Pablo, F. Quiros, A. Rodriguez and J. Vazquez, A general fractional porous medium equation, Comm. Pure Appl. Math. 65 (2012), 1242-1284.

[30] M. del Pino and M. Sáez, On the extinction profile for solutions of $u_{t}=\Delta u^{(n-2) /(n+2)}$, Indiana Univ. Math. J. 50 (2001), no. 1, 611-628.

[31] E. Di Nezza, G. Palatucci and E. Valdinoci, Hitchhiker's guide to the fractional Sobolev spaces, Bull. Sci. Math. 136 (2012), no. 5, 521-573.

[32] J. Dolbeault, Sobolev and Hardy-Littlewood-Sobolev inequalities: duality and fast diffusion, Math. Res. Lett. 18 (2011), no. 6, 1037-1050.

[33] J. Escobar, The Yamabe problem on manifolds with boundary, J. Differential Geom. 35 (1992), no. 1, 21-84.

[34] J. Escobar, Conformal deformation of a Riemannian metric to a scalar flat metric with constant mean curvature on the boundary, Ann. of Math. (2) 136 (1992), no. 1, 1-50.

[35] C. Fefferman and C.R. Graham, Conformal invariants, The mathematical heritage of Élie Cartan (Lyon, 1984). Astérisque 1985, Numero Hors Serie, 95-116.

[36] R. L. Frank and E. Lenzmann, On ground states for the $L^{2}$-critical boson star equation, arXiv:0910.2721v2. 
[37] V. Galaktionov and L. Peletier, Asymptotic behaviour near finite-time extinction for the fast diffusion equation, Arch. Rational Mech. Anal. 139 (1997), no. 1, 83-98.

[38] B. Gidas, W.-M. Ni and L. Nirenberg, Symmetry and related properties via the maximum principle, Comm. Math. Phys. 68 (1979), 209-243.

[39] M. González, R. Mazzeo and Y. Sire, Singular solutions of fractional order conformal Laplacians, J. Geom. Anal. 22 (2012), no. 3, 845-863.

[40] M. González and J. Qing, Fractional conformal Laplacians and fractional Yamabe problems, arXiv:1012.0579v1

[41] C.R. Graham, R. Jenne, L. Mason and G. Sparling, Conformally invariant powers of the Laplacian. I. Existence, J. London Math. Soc. (2) 46 (1992), no. 3, 557-565.

[42] C.R. Graham and M. Zworski, Scattering matrix in conformal geometry, Invent. Math. 152 (2003), 89-118.

[43] P. Guan and G. Wang, A fully nonlinear conformal flow on locally conformally flat manifolds, J. Reine Angew. Math. 557 (2003), 219-238.

[44] R. Hamilton, The Ricci flow on surfaces, pp. 237-262 in : Mathematics and General Relativity, J. Isenberg, ed. Contemp. Math. Vol. 71, AMS, 1988.

[45] Z.-C. Han and Y.Y. Li, The Yamabe problem on manifolds with boundary: existence and compactness results, Duke Math. J. 99 (1999), no. 3, 489-542.

[46] Z.-C. Han and Y.Y. Li, The existence of conformal metrics with constant scalar curvature and constant boundary mean curvature, Comm. Anal. Geom. 8 (2000), no. 4, 809-869.

[47] P.T. Ho, $Q$-curvature flow on $\mathbb{S}^{n}$, Comm. Anal. Geom. 18 (2010), no. 4, 791-820.

[48] M. Jara, Hydrodynamic limit of particle systems with long jumps, arXiv:0805.1326v2.

[49] M. Jara, Nonequilibrium scaling limit for a tagged particle in the simple exclusion process with long jumps, Comm. Pure Appl. Math. 62 (2009), no. 2, 198-214.

[50] M. Jara, T. Komorowski and S. Olla, Limit theorems for additive functionals of a Markov chain, Ann. Appl. Probab. 19 (6) (2009), 2270-2300.

[51] T. Jin, Y.Y. Li and J. Xiong, On a fractional Nirenberg problem, part I: blow up analysis and compactness of solutions, to appear in J. Eur. Math. Soc. (JEMS), arXiv:1111.1332v1.

[52] T. Jin, Y.Y. Li and J. Xiong, On a fractional Nirenberg problem, part II: existence of solutions, preprint, 2012. 
[53] S. Kim and K.-A. Lee, Hölder estimates for singular non-local parabolic equations, J. Funct. Anal. 261 (2011), no. 12, 3482-3518.

[54] M. Kassmann, The classical Harnack inequality fails for nonlocal operators, submitted, available as SFB 611-preprint No. 360.

[55] A. Kochubei, Parabolic pseudodifferential equations, hypersingular integrals and Markov processes (Russian), Izv. Akad. Nauk SSSR Ser. Mat. 52 (1988), no. 5, 909-934, 1118; translation in Math. USSR-Izv. 33 (1989), no. 2, 233-259.

[56] N.V. Krylov, Lectures on elliptic and parabolic equations in Hölder spaces, Graduate Studies in Mathematics, 12. American Mathematical Society, Providence, RI, 1996.

[57] Y.Y. Li, Remark on some conformally invariant integral equations: the method of moving spheres, J. Eur. Math. Soc. (JEMS) 6 (2004), 153-180.

[58] E.H. Lieb, Sharp constants in the Hardy-Littlewood-Sobolev and related inequalities, Ann. of Math. (2) 118 (1983), 349-374.

[59] W.R. Madych and N.M. Rivière, Multipliers of the Hölder classes, J. Funct. Anal. 21 (1976), 369-379.

[60] A. Malchiodi and M. Struwe, $Q$-curvature flow on $\mathbb{S}^{4}$, J. Differential Geom. 73 (2006), no. $1,1-44$.

[61] R. Mazzeo and R. Melrose, Meromorphic extension of the resolvent on complete spaces with asymptotically constant negative curvature, J. Funct. Anal. 75 (1987), 260-310.

[62] R. Mikulevičius and H. Pragarauskas, On the Cauchy problem for certain integrodifferential operators in Sobolev and Hölder spaces. (Lithuanian summary) Liet. Mat. Rink. 32 (1992), no. 2, 299-331; translation in Lithuanian Math. J. 32 (1992), no. 2, $238-264$.

[63] R. Mikulevičius and H. Pragarauskas, On Hölder solutions of the integro-differential Zakai equation, Stochastic Process. Appl. 119 (2009), no. 10, 3319-3355.

[64] R. Mikulevičius and H. Pragarauskas, On the Cauchy problem for integrodifferential operators in Hölder classes and the uniqueness of the martingale problem, arXiv:1103.3492v1.

[65] C. Morpurgo, Sharp inequalities for functional integrals and traces of conformally invariant, Duke Math. J. 114 (2002), 477-553.

[66] L. Nirenberg, A strong maximum principle for parabolic equations, Comm. Pure Appl. Math. 6 (1953), 167-177. 
[67] P. Pavlov and S. Samko, A description of spaces $L_{p}^{\alpha}\left(S_{n-1}\right)$ in terms of spherical hypersingular integrals (Russian), Dokl. Akad. Nauk SSSR 276 (1984), no. 3, 546-550. English translation: Soviet Math. Dokl. 29 (1984), no. 3, 549-553.

[68] J. Qing and D. Raske, On positive solutions to semilinear conformally invariant equations on locally conformally flat manifolds, Int. Math. Res. Not. 2006, Art. ID 94172, 20 pp.

[69] B. Rubin, The inversion of fractional integrals on a sphere, Israel J. Math. 79 (1992), no. $1,47-81$.

[70] H. Schwetlick and M. Struwe, Convergence of the Yamabe flow for "large" energies, J. Reine Angew. Math. 562 (2003), 59-100.

[71] L. Silvestre, Regularity of the obstacle problem for a fractional power of the Laplace operator, Comm. Pure Appl. Math. 60 (2007), 67-112.

[72] L. Simon, Asymptotics for a class of nonlinear evolution equations, with applications to geometric problems, Ann. of Math. (2) 118 (1983), no. 3, 525-571.

[73] E. Stein, Singular integrals and differentiability properties of function, Princeton Mathematical Series, No. 30 Princeton University Press, Princeton, N.J. 1970.

[74] G. Talenti, Best constant in Sobolev inequality, Ann. Mat. Pura Appl. (4) 110 (1976), 353-372.

[75] J. Tan and J. Xiong, A Harnack inequality for fractional Laplace equations with lower order terms, Discrete Contin. Dyn. Syst. 31 (2011), no. 3, 975-983.

[76] $R . Y e$, Global existence and convergence of Yamabe flow, J. Differential Geom. 39 (1994), $35-50$.

Tianling Jin

Department of Mathematics, Rutgers University

110 Frelinghuysen Road, Piscataway, NJ 08854, USA

Email: kingbull@math.rutgers.edu

Jingang Xiong

School of Mathematical Sciences, Beijing Normal University

Beijing 100875, China

and

Department of Mathematics, Rutgers University

110 Frelinghuysen Road, Piscataway, NJ 08854, USA

Email: jxiong@mail.bnu.edu.cn/jxiong@math.rutgers.edu 